\title{
TIME INTEGRATION OF TENSOR TRAINS
}

\author{
CHRISTIAN LUBICH ${ }^{\dagger}$, IVAN V. OSELEDETS $₫$, AND BART VANDEREYCKEN $\S$
}

\begin{abstract}
A robust and efficient time integrator for dynamical tensor approximation in the tensor train or matrix product state format is presented. The method is based on splitting the projector onto the tangent space of the tensor manifold. The algorithm can be used for updating time-dependent tensors in the given data-sparse tensor train / matrix product state format and for computing an approximate solution to high-dimensional tensor differential equations within this data-sparse format. The formulation, implementation and theoretical properties of the proposed integrator are studied, and numerical experiments with problems from quantum molecular dynamics and with iterative processes in the tensor train format are included.
\end{abstract}

Key words. Tensor train, matrix product state, low-rank approximation, time-varying tensors, tensor differential equations, splitting integrator.

AMS subject classifications. 15A18,15A69,65F99,65L05

1. Introduction. There has been much interest lately in the development of data-sparse tensor formats for high-dimensional problems ranging from quantum mechanics to information retrieval; see, e.g., the monograph [5] and references therein. A very promising tensor format is provided by tensor trains (TT) [26, 27], which are also known as matrix product states (MPS) in the theoretical physics literature [29]

In the present paper we deal with the problem of computing an approximation to a time-dependent large tensor $A(t), t_{0} \leq t \leq \bar{t}$ within the TT/MPS format. This includes the situation where the tensor $A(t)$ is known explicitly but in a less datasparse format and we require an approximation of lower complexity. Alternatively, the tensor $A(t)$ could also be defined implicitly as the solution of a tensor differential equation $\dot{A}=F(t, A)$, where ${ }^{\bullet}$ denotes $d / d t$. Such a situation typically arises from a space discretization of a high-dimensional evolutionary partial differential equation.

In both situations, such an approximation can be obtained by the principle of dynamical low-rank: Given an approximation manifold $\mathcal{M}$, the desired time-dependent approximation $Y(t) \in \mathcal{M}$ is computed as

$$
\|\dot{Y}(t)-\dot{A}(t)\|=\min \quad \text { or } \quad\|\dot{Y}(t)-F(t, Y(t))\|=\min ,
$$

where $\dot{A}$ and $F$ are given. This is known as the Dirac-Frenkel time-dependent variational principle in physics; see $[17,18]$. In our case, $\mathcal{M}$ consists of TT/MPS tensors of fixed rank and its manifold structure and tangent space were studied in [12]. For $\|\cdot\|$ the Euclidean norm, the minimizations from above lead to the following differential equations on $\mathcal{M}$ :

$$
\dot{Y}(t)=P_{Y(t)} \dot{A}(t) \quad \text { and } \quad \dot{Y}(t)=P_{Y(t)} F(t, Y(t))
$$

where $P_{Y}$ is the orthogonal projection onto the tangent space of $\mathcal{M}$ at $Y$ (see $\S 3$ for a definition of $P_{Y}$ ). This time-dependent variational principle on fixed-rank TT/MPS

\footnotetext{
${ }^{\dagger}$ Mathematisches Institut, Universität Tübingen, Auf der Morgenstelle 10, D-72076 Tübingen, Germany. (lubich@na.uni-tuebingen.de)

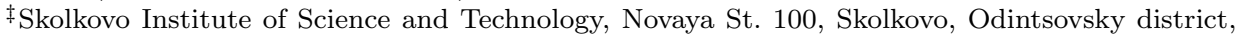
143025 Moscow Region, Russia (i.oseledets@skolkovotech.ru)

『Institute of Numerical Mathematics, Gubkina St. 8, 119333 Moscow, Russia

$\S$ Department of Mathematics, Princeton University, Fine Hall, Princeton NJ 08544, USA. (bartv@math.princeton.edu)
} 
manifolds is studied in [20], where the explicit differential equations are derived and their approximation properties are analyzed. We further refer to [7] for a discussion of time-dependent matrix product state approximations in the physical literature.

A conceptually related, but technically simpler situation arises in the dynamical low-rank approximation of matrices [15]. There, the time-dependent variational principle is applied on manifolds of matrices of a fixed rank, in order to update low-rank approximations to time-dependent large data matrices or to approximate solutions to matrix differential equations by low-rank matrices. The arising differential equations for the low-rank factorization need to be solved numerically, which becomes a challenge in the (often occurring) presence of small singular values in the approximation. While standard numerical integrators such as explicit or implicit Runge-Kutta methods then perform poorly, a novel splitting integrator proposed and studied in [19] shows robustness properties under ill-conditioning that are not shared by any standard numerical integrator. The integrator of [19] is based on splitting the orthogonal projector onto the tangent space of the low-rank matrix manifold. It provides a simple, computationally efficient update of the low-rank factorization in every time step.

In the present paper we extend the projector-splitting integrator of [19] from the matrix case to the TT/MPS case in the time-dependent approximation (1.1).

After collecting the necessary prerequisites on tensor trains / matrix product states in $\S 2$, we study the orthogonal projection onto the tangent space of the fixedrank TT/MPS manifold in $\S 3$. We show that the projector admits an additive decomposition of a simple structure. In $\S 4$ we formulate the algorithm for the splitting integrator based on the decomposition of the projector. In $\S 5$ we show that this integrator inherits from the matrix case an exactness property that gives an indication of the remarkable robustness of the integrator in the presence of small singular values. In $\S 6$ we discuss details of the implementation and present numerical experiments from quantum dynamics and from the application of the integrator to iterative processes in the TT/MPS format.

2. Tensor trains / matrix product states: prerequisites. We present the tensor train or matrix product state formats, together with their normalized representations that we will use throughout the paper. Although our presentation is self-contained, its content is not original and can be found in, e.g, [26, 12].

\subsection{Notation and unfoldings.}

Norm and inner product of tensors. The norm of a tensor $X \in \mathbb{R}^{n_{1} \times \cdots \times n_{d}}$, as considered here, is the Euclidean norm of the vector $\mathbf{x}$ that carries the entries $X\left(\ell_{1}, \ldots, \ell_{d}\right)$ of $X$. The inner product $\langle X, Y\rangle$ of two tensors $X, Y \in \mathbb{R}^{n_{1} \times \cdots \times n_{d}}$ is the Euclidean inner product of the two corresponding vectors $\mathbf{x}$ and $\mathbf{y}$.

Unfolding and reconstruction. The $i$ th unfolding of a tensor $X \in \mathbb{R}^{n_{1} \times \cdots \times n_{d}}$ is the matrix $\mathbf{X}^{\langle i\rangle} \in \mathbb{R}^{\left(n_{1} \cdots n_{i}\right) \times\left(n_{i+1} \cdots n_{d}\right)}$ that aligns all entries $X\left(\ell_{1}, \ldots, \ell_{d}\right)$ with fixed

$\ell_{1}, \ldots, \ell_{i}$ in a row of $\mathbf{X}^{\langle i\rangle}$, and rows and columns are ordered colexicographically. The inverse of unfolding is reconstructing, which we denote as

$$
X=\operatorname{Ten}_{i}\left(\mathbf{X}^{\langle i\rangle}\right)
$$

that is, the tensor $X \in \mathbb{R}^{n_{1} \times \cdots \times n_{d}}$ has the $i$ th unfolding $\mathbf{X}^{\langle i\rangle} \in \mathbb{R}^{\left(n_{1} \ldots n_{i}\right) \times\left(n_{i+1} \ldots n_{d}\right)}$. 
TT/MPS format. A tensor $X \in \mathbb{R}^{n_{1} \times \cdots \times n_{d}}$ is in the TT/MPS format if there exist core tensors $C_{i} \in \mathbb{R}^{r_{i-1} \times n_{i} \times r_{i}}$ with $r_{0}=r_{d}=1$ such that

$$
X\left(\ell_{1}, \ldots, \ell_{d}\right)=\sum_{j_{1}=1}^{r_{1}} \cdots \sum_{j_{d-1}=1}^{r_{d-1}} C_{1}\left(1, \ell_{1}, j_{1}\right) \cdot C_{2}\left(j_{1}, \ell_{2}, j_{2}\right) \cdots C_{d}\left(j_{d-1}, \ell_{d}, 1\right)
$$

for $\ell_{i}=1, \ldots, n_{i}$ and $i=1, \ldots, d$. Equivalently, we have

$$
X\left(\ell_{1}, \ldots, \ell_{d}\right)=\mathbf{C}_{1}\left(\ell_{1}\right) \cdots \mathbf{C}_{d}\left(\ell_{d}\right)
$$

where the $r_{i-1} \times r_{i}$ matrices $\mathbf{C}_{i}\left(\ell_{i}\right)$ are defined as the slices $C_{i}\left(:, \ell_{i},:\right)$.

Observe that $X$ can be parametrized by $\sum_{i=1}^{d} n_{i} r_{i-1} r_{i} \leq d N R^{2}$ degrees of freedom, where $N=\max \left\{n_{i}\right\}$ and $R=\max \left\{r_{i}\right\}$. In high-dimensional applications where TT/MPS tensors are practically relevant, $R$ is constant or only mildly dependent on $d$. Hence for large $d$, one obtains a considerable reduction in the degrees of freedom compared to a general tensor of size $N^{d}$.

Left and right unfoldings. For any core tensor $C_{i} \in \mathbb{R}^{r_{i-1} \times n_{i} \times r_{i}}$, we denote

$$
\mathbf{C}_{i}^{<}=\left[\begin{array}{c}
C_{i}(:, 1,:) \\
\vdots \\
C_{i}\left(:, n_{i},:\right)
\end{array}\right] \in \mathbb{R}^{\left(r_{i-1} n_{i}\right) \times r_{i}}, \quad \mathbf{C}_{i}^{>}=\left[\begin{array}{c}
C_{i}(:,:, 1)^{\top} \\
\vdots \\
C_{i}\left(:,:, r_{i}\right)^{\top}
\end{array}\right] \in \mathbb{R}^{\left(r_{i} n_{i}\right) \times r_{i-1}} .
$$

The matrix $\mathbf{C}_{i}^{<}$is called the left unfolding of $C_{i}$ and $\mathbf{C}_{i}^{>}$is the right unfolding.

$T T / M P S$ rank. We call a vector $\mathbf{r}=\left(1, r_{1}, \ldots, r_{d-1}, 1\right)$ the TT/MPS rank of a tensor $X \in \mathbb{R}^{n_{1} \times \cdots \times n_{d}}$ if

$$
\operatorname{rank} \mathbf{X}^{\langle i\rangle}=r_{i}, \quad(i=1, \ldots, d-1) .
$$

In case $r_{i} \leq \min \left\{\prod_{j=1}^{i} n_{j}, \prod_{j=i+1}^{d} n_{j}\right\}$, this implies that $X$ can be represented in the TT/MPS format with core tensors $C_{i} \in \mathbb{R}^{r_{i-1} \times n_{i} \times r_{i}}$ of full multi-linear rank, that is,

$$
\operatorname{rank} \mathbf{C}_{i}^{<}=r_{i} \quad \text { and } \quad \operatorname{rank} \mathbf{C}_{i}^{>}=r_{i-1}, \quad(i=1, \ldots, d) .
$$

In addition, it is known (see [12, Lem. 4]) that for fixed $\mathbf{r}$ such a full-rank condition on the core tensors implies that the set

$$
\mathcal{M}=\left\{X \in \mathbb{R}^{n_{1} \times \cdots \times n_{d}}: \text { TT/MPS rank of } X \text { is } \mathbf{r}\right\}
$$

is a smooth embedded submanifold in $\mathbb{R}^{n_{1} \times \cdots \times n_{d}}$.

Partial products. Define the left partial product $X_{\leq i} \in \mathbb{R}^{n_{1} \times \cdots \times n_{i} \times r_{i}}$ as

$$
X_{\leq i}\left(\ell_{1}, \ldots, \ell_{i},:\right)=\mathbf{C}_{1}\left(\ell_{1}\right) \cdots \mathbf{C}_{i}\left(\ell_{i}\right)
$$

and the right partial product $X_{\geq i+1} \in \mathbb{R}^{r_{i} \times n_{i+1} \times \cdots \times n_{d}}$ as

$$
X_{\geq i+1}\left(:, \ell_{i+1}, \ldots, \ell_{d}\right)=\mathbf{C}_{i+1}\left(\ell_{i+1}\right) \cdots \mathbf{C}_{d}\left(\ell_{d}\right) .
$$

See also Fig. 2.1(a) for their graphical representation in terms of a tensor network.

Let a particular unfolding of each of these partial products be denoted as

$$
\mathbf{X}_{\leq i} \in \mathbb{R}^{\left(n_{1} \cdots n_{i}\right) \times r_{i}}, \quad \mathbf{X}_{\geq i+1} \in \mathbb{R}^{\left(n_{i+1} \cdots n_{d}\right) \times r_{i}} .
$$


(a)

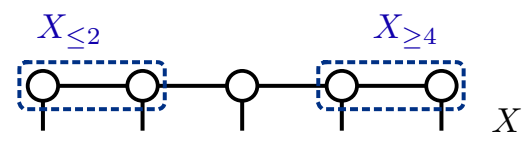

(b)

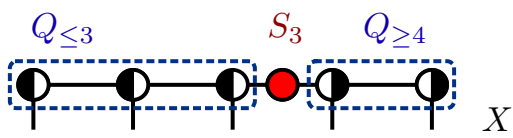

Fig. 2.1. A 5 dimensional TT/MPS tensor X. Panel (a) indicates specific left and right partial products of $X$. Panel (b) depicts the third recursive $S V D$ of $X$. Observe that left and right orthogonalized cores are denoted using $($ and respectively.

The elementwise relation $X\left(\ell_{1}, \ldots, \ell_{d}\right)=X_{\leq i}\left(\ell_{1}, \ldots, \ell_{i},:\right) X_{\geq i+1}\left(:, \ell_{i+1}, \ldots, \ell_{d}\right)$ then translates into

$$
\mathbf{X}^{\langle i\rangle}=\mathbf{X}_{\leq i} \mathbf{X}_{\geq i+1}^{\top} .
$$

Recursive construction. We note the recurrence relations

$$
\mathbf{X}_{\leq i}=\left(\mathbf{I}_{n_{i}} \otimes \mathbf{X}_{\leq i-1}\right) \mathbf{C}_{i}^{<} \quad(i=1, \ldots, d)
$$

starting from $\mathbf{X}_{\leq 0}=1$, and

$$
\mathbf{X}_{\geq i}=\left(\mathbf{X}_{\geq i+1} \otimes \mathbf{I}_{n_{i}}\right) \mathbf{C}_{i}^{>} \quad(i=1, \ldots, d)
$$

with $\mathbf{X}_{\geq d+1}=1$. Here $\otimes$ denotes the standard Kronecker product.

Combining the above formulas we note

$$
\mathbf{X}^{\langle i\rangle}=\left(\mathbf{I}_{n_{i}} \otimes \mathbf{X}_{\leq i-1}\right) \mathbf{C}_{i}^{<} \mathbf{X}_{\geq i+1}^{\top},
$$

which will be an important formula later. Using the recurrence relations for $\mathbf{X}_{\geq i}$ we also obtain

$$
\mathbf{X}^{\langle i-1\rangle}=\mathbf{X}_{\leq i-1} \mathbf{C}_{i}^{>\top}\left(\mathbf{X}_{\geq i+1} \otimes \mathbf{I}_{n_{i}}\right)^{\top},
$$

which together with the previous formula allows us to pass from the $(i-1)$ th to the $i$ th unfolding.

2.2. Left and right orthogonalizations. Thanks to the recursive relations (2.2) and (2.3), it is possible to compute the QR decompositions of the matrices $\mathbf{X}_{\leq i}$ and $\mathbf{X}_{\geq i}$ efficiently.

Let us explain the case for $\mathbf{X}_{\leq i}$ in detail. First, compute a QR factorization (the $<$ in $\mathbf{Q}_{1}^{<}$is just notational for now but will become clear in $\S 2.3$ ),

$$
\mathbf{X}_{\leq 1}=\mathbf{C}_{1}^{<}=\mathbf{Q}_{1}^{<} \mathbf{R}_{1}, \quad \text { with } \quad \mathbf{Q}_{1}^{<\top} \mathbf{Q}_{1}^{<}=\mathbf{I}_{r_{1}}, \mathbf{Q}_{1}^{<} \in \mathbb{R}^{n_{1} \times r_{1}}, \mathbf{R}_{1} \in \mathbb{R}^{r_{1} \times r_{1}},
$$

and insert it into the recurrence relation (2.2) to obtain

$$
\mathbf{X}_{\leq 2}=\left(\mathbf{I}_{n_{2}} \otimes \mathbf{Q}_{1}^{<} \mathbf{R}_{1}\right) \mathbf{C}_{2}^{<}=\left(\mathbf{I}_{n_{2}} \otimes \mathbf{Q}_{1}^{<}\right)\left(\mathbf{I}_{n_{2}} \otimes \mathbf{R}_{1}\right) \mathbf{C}_{2}^{<} .
$$

Next, make another QR decomposition

$$
\left(\mathbf{I}_{n_{2}} \otimes \mathbf{R}_{1}\right) \mathbf{C}_{2}^{<}=\mathbf{Q}_{2}^{<} \mathbf{R}_{2}, \quad \text { with } \quad \mathbf{Q}_{2}^{<\top} \mathbf{Q}_{2}^{<}=\mathbf{I}_{r_{2}}, \mathbf{Q}_{2}^{<} \in \mathbb{R}^{\left(r_{1} n_{2}\right) \times r_{2}}, \mathbf{R}_{2} \in \mathbb{R}^{r_{2} \times r_{2}},
$$

so that we have obtained a $\mathrm{QR}$ decomposition of

$$
\mathbf{X}_{\leq 2}=\mathbf{Q}_{\leq 2} \mathbf{R}_{2} \quad \text { with } \quad \mathbf{Q}_{\leq 2}=\left(\mathbf{I}_{n_{2}} \otimes \mathbf{Q}_{1}^{<}\right) \mathbf{Q}_{2}^{<} .
$$


These orthogonalizations can be continued in the same way for $i=2,3, \ldots$. Putting $\mathbf{Q}_{\leq 0}=1$, we have obtained for each $i=1, \ldots, d$ the $\mathrm{QR}$ decompositions

$$
\mathbf{X}_{\leq i}=\mathbf{Q}_{\leq i} \mathbf{R}_{i} \quad \text { with } \quad \mathbf{Q}_{\leq i}=\left(\mathbf{I}_{n_{i}} \otimes \mathbf{Q}_{\leq i-1}\right) \mathbf{Q}_{i}^{<}
$$

where the matrices $\mathbf{Q}_{i}^{<} \in \mathbb{R}^{\left(r_{i-1} n_{i}\right) \times r_{i}}$ and $\mathbf{R}_{i} \in \mathbb{R}^{r_{i} \times r_{i}}$ are obtained recursively from QR decompositions of lower-dimensional matrices $\left(\mathbf{I}_{n_{i}} \otimes \mathbf{R}_{i-1}\right) \mathbf{C}_{i}^{<}=\mathbf{Q}_{i}^{<} \mathbf{R}_{i}$. We call the left partial product $\mathbf{X}_{\leq i}$ in that case left-orthogonalized.

In a completely analogous way, we can obtain a right-orthogonalized $\mathbf{X}_{\geq i}$ as follows. Denote $\mathbf{Q}_{\geq d+1}=1$. Then, starting with $\mathbf{X}_{\geq d}=\mathbf{C}_{d}^{>}=\mathbf{Q}_{d}^{>} \mathbf{R}_{d}$, we can use (2.3) to obtain the $\mathrm{QR}$ decompositions

$$
\mathbf{X}_{\geq i}=\mathbf{Q}_{\geq i} \mathbf{R}_{i} \quad \text { with } \quad \mathbf{Q}_{\geq i}=\left(\mathbf{Q}_{\geq i+1} \otimes \mathbf{I}_{n_{i}}\right) \mathbf{Q}_{i}^{>},
$$

where the matrices $\mathbf{Q}_{i}^{>} \in \mathbb{R}^{\left(r_{i} n_{i}\right) \times r_{i-1}}$ and $\mathbf{R}_{i} \in \mathbb{R}^{r_{i-1} \times r_{i-1}}$ are recursively obtained from $\left(\mathbf{R}_{i+1} \otimes \mathbf{I}_{n_{i}}\right) \mathbf{C}_{i}^{>}=\mathbf{Q}_{i}^{>} \mathbf{R}_{i}$. We remark that these $\mathbf{R}_{i}$ are in general different than those obtained while orthogonalizing from the left.

Observe that when $\mathbf{X}_{\leq i}$ is left-orthogonalized, then so is $\mathbf{X}_{\leq j}$ for any $j<i$. Since $\mathbf{X}_{\leq d}=\mathbf{X}^{\langle d\rangle}$, we call $X$ left orthogonal if $\mathbf{X}_{\leq d}$ is left-orthogonalized. As is evident from Fig. 2.1, such a left orthogonal $X$ is recursively computed by modifying the cores $C_{i}$ from left to right during a so-called forward sweep. Likewise, we call $X$ right orthogonal if $\mathbf{X}_{\geq 1}=\mathbf{X}^{\langle 1\rangle}$ is right-orthogonalized which is obtained by a backward sweep from right to left.

2.3. Recursive SVD. Suppose that $\mathbf{X}_{\leq i}=\mathbf{Q}_{\leq i} \mathbf{R}_{i}$ and $\mathbf{X}_{\geq i+1}=\mathbf{Q}_{>i+1} \mathbf{R}_{i+1}$ are $\mathrm{QR}$ decompositions obtained from left and right orthogonalizations, we then have the following SVD-like decomposition

$$
\mathbf{X}^{\langle i\rangle}=\mathbf{Q}_{\leq i} \mathbf{S}_{i} \mathbf{Q}_{\geq i+1}^{\top}, \quad \text { with } \quad \mathbf{S}_{i}=\mathbf{R}_{i} \mathbf{R}_{i+1}^{\top} \in \mathbb{R}^{r_{i} \times r_{i}} .
$$

The matrix $\mathbf{S}_{i}$ can be chosen diagonal, although we do not insist that it is. Since the orthonormal matrices $\mathbf{Q}_{\leq i}$ and $\mathbf{Q}_{\geq i+1}$ satisfy the recursive relations as explained before, we call (2.7) a recursive $S V D$ of $\mathbf{X}^{\langle i\rangle}$, or the $i$ th recursive SVD of $X$. The graphical representation of such a recursive SVD is depicted in Fig. 2.1(b).

This recursiveness can be used for the SVD of $\mathbf{X}^{\langle i+1\rangle}$. By (2.6), we can write

$$
\mathbf{X}^{\langle i\rangle}=\left(\mathbf{Q}_{\leq i} \mathbf{S}_{i}\right) \mathbf{Q}_{i+1}^{>\boldsymbol{\top}}\left(\mathbf{Q}_{\geq i+2} \otimes \mathbf{I}_{n_{i+1}}\right)^{\boldsymbol{\top}}
$$

To obtain a decomposition of $\mathbf{X}^{\langle i+1\rangle}$ by means of the relations (2.5) and (2.4), we identify (2.8) with (2.5) (hence, $i-1$ takes the role of $i$ and $\mathbf{C}_{i}^{>\top}$ that of $\mathbf{Q}_{i+1}^{>\top}$ ). The corresponding expression for (2.4) then becomes

$$
\mathbf{X}^{\langle i+1\rangle}=\left(\mathbf{I}_{n_{i+1}} \otimes \mathbf{Q}_{\leq i} \mathbf{S}_{i}\right) \mathbf{Q}_{i+1}^{<} \mathbf{Q}_{\geq i+2}^{\top},
$$

which we can also write as

$$
\mathbf{X}^{\langle i+1\rangle}=\left(\mathbf{I}_{n_{i+1}} \otimes \mathbf{Q}_{\leq i}\right)\left(\mathbf{I}_{n_{i+1}} \otimes \mathbf{S}_{i}\right) \mathbf{Q}_{i+1}^{<} \mathbf{Q}_{\geq i+2}^{\top} .
$$

Hence, after a QR decomposition

$$
\left(\mathbf{I}_{n_{i+1}} \otimes \mathbf{S}_{i}\right) \mathbf{Q}_{i+1}^{<}=\overline{\mathbf{Q}}_{i+1}^{<} \overline{\mathbf{S}}_{i+1},
$$


we obtain the $(i+1)$ th recursive SVD

$$
\mathbf{X}^{\langle i+1\rangle}=\overline{\mathbf{Q}}_{\leq i+1} \overline{\mathbf{S}}_{i+1} \mathbf{Q}_{\geq i+2}^{\top} \quad \text { with } \quad \overline{\mathbf{Q}}_{\leq i+1}=\left(\mathbf{I}_{n_{i+1}} \otimes \mathbf{Q}_{\leq i}\right) \overline{\mathbf{Q}}_{i+1}^{<} .
$$

A similar relation holds between $\mathbf{X}^{\langle i\rangle}$ and $\mathbf{X}^{\langle i-1\rangle}$. Let

$$
\mathbf{X}^{\langle i\rangle}=\mathbf{Q}_{\leq i} \mathbf{S}_{i} \mathbf{Q}_{\geq i+1}^{\boldsymbol{\top}}=\left(\mathbf{I}_{n_{i}} \otimes \mathbf{Q}_{\leq i-1}\right) \mathbf{Q}_{i}^{<}\left(\mathbf{Q}_{\geq i+1} \mathbf{S}_{i}^{\boldsymbol{\top}}\right)^{\boldsymbol{\top}},
$$

then using the $\mathrm{QR}$ decomposition

$$
\left(\mathbf{S}_{i}^{\top} \otimes \mathbf{I}_{n_{i}}\right) \mathbf{Q}_{i}^{>}=\overline{\mathbf{Q}}_{i}^{>} \overline{\mathbf{S}}_{i}^{\top}
$$

we can write

$$
\mathbf{X}^{\langle i-1\rangle}=\mathbf{Q}_{\leq i-1} \overline{\mathbf{S}}_{i} \overline{\mathbf{Q}}_{\geq i}^{\top} \quad \text { where } \quad \overline{\mathbf{Q}}_{\geq i}=\left(\mathbf{Q}_{\geq i+1} \otimes \mathbf{I}_{n_{i}}\right) \overline{\mathbf{Q}}_{i}^{>} .
$$

3. Orthogonal projection onto the tangent space. Let $\mathcal{M}$ be the embedded manifold of tensors of a given TT/MPS rank $\mathbf{r}$; see (2.1). In this section, we derive an explicit formula for the orthogonal projection onto the tangent space $T_{X} \mathcal{M}$ at $X \in \mathcal{M}$,

$$
P_{X}: \mathbb{R}^{n_{1} \times \cdots \times n_{d}} \rightarrow T_{X} \mathcal{M}
$$

With the Euclidean inner product, the projection $P_{X}(Z)$ for arbitrary $Z \in \mathbb{R}^{n_{1} \times \cdots \times n_{d}}$ has the following equivalent variational definition:

$$
\left\langle P_{X}(Z), \delta X\right\rangle=\langle Z, \delta X\rangle \quad \forall \delta X \in T_{X} \mathcal{M} .
$$

Before we state the theorem, we recall a useful parametrization of $T_{X} \mathcal{M}$ as introduced in [12]. Let $X \in \mathcal{M}$ be left orthogonal, that is, in the decompositions

$$
\mathbf{X}^{\langle i\rangle}=\left(\mathbf{I}_{n_{i}} \otimes \mathbf{X}_{\leq i-1}\right) \mathbf{C}_{i}^{<} \mathbf{X}_{\geq i+1}^{\top},
$$

the matrices satisfy for all $i=1, \ldots, d-1$

$$
\mathbf{X}_{\leq i}^{\top} \mathbf{X}_{\leq i}=\mathbf{I}_{r_{i}} \text { and } \mathbf{C}_{i}^{<\boldsymbol{\top}} \mathbf{C}_{i}^{<}=\mathbf{I}_{r_{i}}
$$

Define then for $i=1, \ldots, d-1$ the subspaces

$$
\mathcal{V}_{i}=\left\{\operatorname{Ten}_{i}\left[\left(\mathbf{I}_{n_{i}} \otimes \mathbf{X}_{\leq i-1}\right) \delta \mathbf{C}_{i}^{<} \mathbf{X}_{\geq i+1}^{\boldsymbol{\top}}\right]: \delta C_{i} \in \mathbb{R}^{r_{i-1} \times n_{i} \times r_{i}} \text { and } \mathbf{C}_{i}^{<\boldsymbol{\top}} \delta \mathbf{C}_{i}^{<}=\mathbf{0}\right\}
$$

and also the subspace

$$
\mathcal{V}_{d}=\left\{\operatorname{Ten}_{d}\left[\left(\mathbf{I}_{n_{d}} \otimes \mathbf{X}_{\leq d-1}\right) \delta \mathbf{C}_{d}^{<}\right]: \delta C_{d} \in \mathbb{R}^{r_{d-1} \times n_{d} \times r_{d}}\right\} .
$$

Observe that these subspaces represent the first-order variations in $C_{i}$ in all the representations (3.1) together with the so-called gauge conditions $\mathbf{C}_{i}^{<\boldsymbol{\top}} \delta \mathbf{C}_{i}^{<}=\mathbf{0}$ when $i \neq d$; there is no gauge condition for $i=d$. Now, [12, Thm. 4] states that

$$
T_{X} \mathcal{M}=\mathcal{V}_{1} \oplus \mathcal{V}_{2} \oplus \cdots \oplus \mathcal{V}_{d}
$$

In other words, every $\delta X \in T_{X} \mathcal{M}$ admits the unique orthogonal ${ }^{1}$ decomposition

$$
\delta X=\sum_{i=1}^{d} \delta X_{i}, \quad \text { with } \quad \delta X_{i} \in \mathcal{V}_{i}, \quad \text { and } \quad\left\langle\delta X_{i}, \delta X_{j}\right\rangle=\delta_{i j}
$$

\footnotetext{
${ }^{1}$ The orthogonality of the $\mathcal{V}_{i}$ spaces is only implicitly present in [12, Thm. 4]; it is however not difficult to prove it explicitly thanks to the left-orthogonalization and the gauge conditions.
} 
Now we are ready to state the formula for $P_{X}$. It uses the orthogonal projections onto the range of $\mathbf{X}_{\leq i}$, denoted as $\mathbf{P}_{\leq i}$, and onto the range of $\mathbf{X}_{\geq i}$, denoted as $\mathbf{P}_{\geq i}$. With the QR decompositions $\mathbf{X}_{\leq i}=\mathbf{Q}_{\leq i} \mathbf{R}_{i}$ and $\mathbf{X}_{\geq i}=\mathbf{Q}_{\geq i} \mathbf{R}_{i}^{\prime}$, these projections become

$$
\mathbf{P}_{\leq i}=\mathbf{Q}_{\leq i} \mathbf{Q}_{\leq i}^{\top}, \quad \text { and } \quad \mathbf{P}_{\geq i}=\mathbf{Q}_{\geq i} \mathbf{Q}_{\geq i}^{\top} .
$$

We set $\mathbf{P}_{\leq 0}=1$ and $\mathbf{P}_{\geq d+1}=1$.

THEOREM 3.1. Let $\mathcal{M}$ be the manifold of fixed rank TT/MPS tensors. Then, the orthogonal projection onto the tangent space of $\mathcal{M}$ at $X \in \mathcal{M}$ is given by

$$
\begin{aligned}
P_{X}(Z)= & \sum_{i=1}^{d-1} \operatorname{Ten}_{i}\left[\left(\mathbf{I}_{n_{i}} \otimes \mathbf{P}_{\leq i-1}\right) \mathbf{Z}^{\langle i\rangle} \mathbf{P}_{\geq i+1}-\mathbf{P}_{\leq i} \mathbf{Z}^{\langle i\rangle} \mathbf{P}_{\geq i+1}\right] \\
& +\operatorname{Ten}_{d}\left[\left(\mathbf{I}_{n_{d}} \otimes \mathbf{P}_{\leq d-1}\right) \mathbf{Z}^{\langle d\rangle}\right]
\end{aligned}
$$

for any $Z \in \mathbb{R}^{n_{1} \times \cdots \times n_{d}}$.

Proof. We assume that $X$ is given by (3.1). For given $Z \in \mathbb{R}^{n_{1} \times \cdots \times n_{d}}$, we aim to determine $\delta U=P_{X}(Z) \in T_{X} \mathcal{M}$ such that

$$
\langle\delta U, \delta X\rangle=\langle Z, \delta X\rangle \quad \forall \delta X \in T_{X} \mathcal{M} .
$$

Writing $\delta U=\sum_{j=1}^{d} \delta U_{j}$ with $\delta U_{j} \in \mathcal{V}_{j}$, this means that we need to determine matrices $\delta \mathbf{B}_{j}^{<}$in the unfoldings

$$
\delta \mathbf{U}_{j}^{\langle j\rangle}=\left(\mathbf{I}_{n_{j}} \otimes \mathbf{X}_{\leq j-1}\right) \delta \mathbf{B}_{j}^{<} \mathbf{X}_{\geq j+1}^{\boldsymbol{\top}},
$$

such that the gauge conditions are satisfied

$$
\mathbf{C}_{j}^{<\boldsymbol{T}} \delta \mathbf{B}_{j}^{<}=\mathbf{0} \quad(j=1, \ldots, d-1) .
$$

Fix an $i$ between 1 and $d$. Since $\mathcal{V}_{i}$ is orthogonal to $\mathcal{V}_{j}$ when $j \neq i$, choosing any $\delta X=\delta X_{i} \in \mathcal{V}_{i}$ in (3.2) implies

$$
\left\langle\delta U_{i}, \delta X_{i}\right\rangle=\left\langle Z, \delta X_{i}\right\rangle \quad \forall \delta X_{i} \in \mathcal{V}_{i} .
$$

Parametrize $\delta X_{i} \in \mathcal{V}_{i}$ as

$$
\delta \mathbf{X}_{i}^{\langle i\rangle}=\left(\mathbf{I}_{n_{i}} \otimes \mathbf{X}_{\leq i-1}\right) \delta \mathbf{C}_{i}^{<} \mathbf{X}_{\geq i+1}^{\top}
$$

with $\delta \mathbf{C}_{i}^{<}$satisfying the gauge condition for $i \neq d$. Then, the left-hand side of (3.3) becomes

$$
\begin{aligned}
\left\langle\delta U_{i}, \delta X_{i}\right\rangle & =\left\langle\delta \mathbf{U}_{i}^{\langle i\rangle}, \delta \mathbf{X}_{i}^{\langle i\rangle}\right\rangle \\
& =\left\langle\left(\mathbf{I}_{n_{i}} \otimes \mathbf{X}_{\leq i-1}\right) \delta \mathbf{B}_{i}^{<} \mathbf{X}_{\geq i+1}^{\boldsymbol{\top}},\left(\mathbf{I}_{n_{i}} \otimes \mathbf{X}_{\leq i-1}\right) \delta \mathbf{C}_{i}^{<} \mathbf{X}_{\geq i+1}^{\boldsymbol{\top}}\right\rangle \\
& =\left\langle\delta \mathbf{B}_{i}^{<} \mathbf{X}_{\geq i+1}^{\top} \mathbf{X}_{\geq i+1}, \delta \mathbf{C}_{i}^{<}\right\rangle
\end{aligned}
$$

since $X$ is left orthogonal. Likewise, for the right-hand side we get

$$
\begin{aligned}
\left\langle Z, \delta X_{i}\right\rangle & =\left\langle\mathbf{Z}^{\langle i\rangle}, \delta \mathbf{X}_{i}^{\langle i\rangle}\right\rangle \\
& =\left\langle\left(\mathbf{I}_{n_{i}} \otimes \mathbf{X}_{\leq i-1}\right)^{\top} \mathbf{Z}^{\langle i\rangle} \mathbf{X}_{\geq i+1}, \delta \mathbf{C}_{i}^{<}\right\rangle
\end{aligned}
$$


Hence, for all matrices $\delta \mathbf{C}_{i}^{<}$satisfying the gauge conditions, we must have

$$
\left\langle\delta \mathbf{B}_{i}^{<} \mathbf{X}_{\geq i+1}^{\top} \mathbf{X}_{\geq i+1}, \delta \mathbf{C}_{i}^{<}\right\rangle=\left\langle\left(\mathbf{I}_{n_{i}} \otimes \mathbf{X}_{\leq i-1}\right)^{\top} \mathbf{Z}^{\langle i\rangle} \mathbf{X}_{\geq i+1}, \delta \mathbf{C}_{i}^{<}\right\rangle
$$

which implies, with $\mathbf{P}_{i}^{<}$the orthogonal projector onto the range of $\mathbf{C}_{i}^{<}$for $i=$ $1, \ldots, d-1$ and with $\mathbf{P}_{i}^{<}=\mathbf{0}$ for $i=d$,

$$
\delta \mathbf{B}_{i}^{<}=\left(\mathbf{I}_{i}-\mathbf{P}_{i}^{<}\right)\left(\mathbf{I}_{n_{i}} \otimes \mathbf{X}_{\leq i-1}\right)^{\top} \mathbf{Z}^{\langle i\rangle} \mathbf{X}_{\geq i+1}\left(\mathbf{X}_{\geq i+1}^{\top} \mathbf{X}_{\geq i+1}\right)^{-1}
$$

where $\mathbf{I}_{i}=\mathbf{I}_{n_{i} r_{i-1}}$. Inserting this expression into the formula for $\delta \mathbf{U}_{i}^{\langle i\rangle}$ gives us

$$
\delta \mathbf{U}_{i}^{\langle i\rangle}=\left(\mathbf{I}_{n_{i}} \otimes \mathbf{X}_{\leq i-1}\right)\left(\mathbf{I}_{i}-\mathbf{P}_{i}^{<}\right)\left(\mathbf{I}_{n_{i}} \otimes \mathbf{X}_{\leq i-1}\right)^{\top} \mathbf{Z}^{\langle i\rangle} \mathbf{X}_{\geq i+1}\left(\mathbf{X}_{\geq i+1}^{\top} \mathbf{X}_{\geq i+1}\right)^{-1} \mathbf{X}_{\geq i+1}^{\top}
$$

Since $\mathbf{P}_{\leq i-1}=\mathbf{X}_{\leq i-1} \mathbf{X}_{\leq i-1}^{\top}, \mathbf{P}_{\leq i}=\left(\mathbf{I}_{n_{i}} \otimes \mathbf{X}_{\leq i-1}\right) \mathbf{P}_{i}^{<}\left(\mathbf{I}_{n_{i}} \otimes \mathbf{X}_{\leq i-1}\right)^{\top}$ and $\mathbf{P}_{\geq i+1}=$ $\mathbf{X}_{\geq i+1}\left(\mathbf{X}_{\geq i+1}^{\top} \mathbf{X}_{\geq i+1}\right)^{-1} \mathbf{X}_{\geq i+1}^{\top}$, this simplifies to

$$
\begin{aligned}
\delta \mathbf{U}_{i}^{\langle i\rangle} & =\left(\mathbf{I}_{n_{i}} \otimes \mathbf{P}_{\leq i-1}-\mathbf{P}_{\leq i}\right) \mathbf{Z}^{\langle i\rangle} \mathbf{P}_{\geq i+1} \quad(i=1, \ldots, d-1), \\
\delta \mathbf{U}_{d}^{\langle d\rangle} & =\left(\mathbf{I}_{n_{d}} \otimes \mathbf{P}_{\leq d-1}\right) \mathbf{Z}^{\langle i\rangle} .
\end{aligned}
$$

Now $\delta U=\sum_{i=1}^{d} \delta U_{i}$ satisfies the projection condition (3.2). $\square$

Although the formula in Theorem 3.1 lends itself well to practical implementation, its cumbersome notation is a nuisance. We therefore introduce a simpler notation for the forthcoming derivations.

Corollary 3.2. For $i=0, \ldots, d+1$, define the orthogonal projectors

$$
\begin{aligned}
& P_{\leq i}: \mathbb{R}^{n_{1} \times \cdots \times n_{d}} \rightarrow T_{X} \mathcal{M}, Z \mapsto \operatorname{Ten}_{i}\left(\mathbf{P}_{\leq i} \mathbf{Z}^{\langle i\rangle}\right) \\
& P_{\geq i}: \mathbb{R}^{n_{1} \times \cdots \times n_{d}} \rightarrow T_{X} \mathcal{M}, Z \mapsto \operatorname{Ten}_{i-1}\left(\mathbf{Z}^{\langle i-1\rangle} \mathbf{P}_{\geq i}\right) .
\end{aligned}
$$

Then, the projector $P_{X}$ in Theorem 3.1 satisfies

$$
P_{X}=\sum_{i=1}^{d-1}\left(P_{\leq i-1} P_{\geq i+1}-P_{\leq i} P_{\geq i+1}\right)+P_{\leq d-1} P_{\geq d+1} .
$$

In addition, $P_{\leq i}$ and $P_{\geq j}$ commute for $i<j$.

Proof. The fact that $P_{\leq i}$ commutes with $P_{\geq j}$ follows from the observation that for any $Z \in \mathbb{R}^{n_{1} \times \cdots \times n_{d}}, P_{\leq i}(Z)$ acts on the rows of $\mathbf{Z}^{\langle i\rangle}$ - and hence also on the rows of $\mathbf{Z}^{\langle j\rangle}$ - while $P_{\geq j}(Z)$ acts on the columns of $\mathbf{Z}^{\langle j\rangle}$.

To write $P_{X}$ using the new notation, we need to work out the term

$$
P_{\geq i+1}\left(P_{\leq i-1}(Z)\right)=P_{\geq i+1}\left[\operatorname{Ten}_{i-1}\left(\mathbf{P}_{\leq i-1} \mathbf{Z}^{\langle i-1\rangle}\right)\right]=\operatorname{Ten}_{i}\left[\mathbf{Y}^{\langle i\rangle} \mathbf{P}_{\geq i+1}\right]
$$

with $Y=\operatorname{Ten}_{i-1}\left(\mathbf{P}_{\leq i-1} \mathbf{Z}^{\langle i-1\rangle}\right)$. Denote the mode-1 matricization of a tensor by .(1); see $[16, \S 2.4]$ for a definition. Then, define the tensors $\widehat{Z}$ and $\widehat{Y}$, both of size $\left(n_{1} \cdots n_{i-1}\right) \times n_{i} \times\left(n_{i+1} \cdots n_{d}\right)$, such that $\widehat{\mathbf{Z}}^{(1)}=\mathbf{Z}^{\langle i-1\rangle}$ and $\widehat{\mathbf{Y}}^{(1)}=\mathbf{Y}^{\langle i-1\rangle}$. In addition, let $\times_{1}$ denote the mode-1 multilinear product of a tensor with a matrix; see $[16, \S 2.5]$. Then, using $[16$, p. 426$]$ to compute matricizations of multilinear products, we get

$$
\left(\widehat{Z} \times{ }_{1} \mathbf{P}_{\leq i-1}\right)^{(1)}=\mathbf{P}_{\leq i-1} \widehat{\mathbf{Z}}^{(1)}=\mathbf{P}_{\leq i-1} \mathbf{Z}^{\langle i-1\rangle}=\mathbf{Y}^{\langle i-1\rangle}=\widehat{\mathbf{Y}}^{(1)} .
$$


Hence, we see that $\widehat{Y}=\widehat{Z} \times{ }_{1} \mathbf{P}_{\leq i-1}$. Using the notation ${ }^{(1,2)}={ }^{(3) T}$ (see again [16, $\S 2.4])$, we obtain

$$
\widehat{\mathbf{Y}}^{(1,2)}=\left(\widehat{Z} \times{ }_{1} \mathbf{P}_{\leq i-1}\right)^{(1,2)}=\left(\mathbf{I}_{n_{i}} \otimes \mathbf{P}_{\leq i-1}\right) \widehat{\mathbf{Z}}^{(1,2)} .
$$

Now, observe that because of the colexicographical ordering of unfoldings and matricizations, we have $\widehat{\mathbf{Z}}^{(1,2)}=\mathbf{Z}^{\langle i\rangle}$ and $\widehat{\mathbf{Y}}^{(1,2)}=\mathbf{Y}^{\langle i\rangle}$ and this gives

$$
P_{\geq i+1}\left(P_{\leq i-1}(Z)\right)=\operatorname{Ten}_{i}\left[\mathbf{Y}^{\langle i\rangle} \mathbf{P}_{\geq i+1}\right]=\operatorname{Ten}_{i}\left[\left(\mathbf{I}_{n_{i}} \otimes \mathbf{P}_{\leq i-1}\right) \mathbf{Z}^{\langle i\rangle} \mathbf{P}_{\geq i+1}\right] .
$$

The term $P_{\leq i} P_{\geq i+1}$ is straightforward, and this finishes the proof. $\square$

4. Projector-splitting integrator. We now consider the main topic of this paper: a numerical integrator for the dynamical TT/MPS approximation

$$
\dot{Y}(t)=P_{Y(t)}(\dot{A}(t)), \quad Y\left(t_{0}\right)=Y_{0} \in \mathcal{M}
$$

of a given time-dependent tensor $A(t) \in \mathbb{R}^{n_{1} \times \cdots \times n_{d}}$.

Our integrator is a Lie-Trotter splitting of the vector field $P_{Y}(\dot{A})$. The splitting itself is suggested by the sum in Corollary 3.2: using $Y$ in the role of $X$, we can write

$$
P_{Y}(\dot{A})=P_{1}^{+}(\dot{A})-P_{1}^{-}(\dot{A})+P_{2}^{+}(\dot{A})-P_{2}^{-}(\dot{A})+\cdots-P_{d-1}^{-}(\dot{A})+P_{d}^{+}(\dot{A})
$$

with the orthogonal projectors

$$
\begin{array}{lr}
P_{i}^{+}(Z)=P_{\leq i-1} P_{\geq i+1}(Z)=\operatorname{Ten}_{i}\left[\left(\mathbf{I}_{n_{i}} \otimes \mathbf{P}_{\leq i-1}\right) \mathbf{Z}^{\langle i\rangle} \mathbf{P}_{\geq i+1}\right], & (1 \leq i \leq d) \\
P_{i}^{-}(Z)=P_{\leq i} P_{\geq i+1}(Z)=\operatorname{Ten}_{i}\left[\mathbf{P}_{\leq i} \mathbf{Z}^{\langle i\rangle} \mathbf{P}_{\geq i+1}\right], & (1 \leq i \leq d-1)
\end{array}
$$

By standard theory (see, e.g., [8, II.5]), any splitting of this sum results in a firstorder integrator, and composing it with the adjoint gives a second-order integrator, also known as the Strang splitting. Somewhat remarkably, we shall show in Thm. 4.1 that these split differential equations can be solved in closed form. Furthermore, if they are solved from left to right (or from right to left), the whole scheme can be implemented very efficiently.

4.1. Abstract formulation and closed-form solutions. Let $t_{1}-t_{0}>0$ be the step size. One full step of the splitting integrator solves in consecutive order the following initial value problems over the time interval $\left[t_{0}, t_{1}\right]$ :

$$
\begin{array}{rlrl}
\dot{Y}_{1}^{+} & =+P_{1}^{+}(\dot{A}), & & Y_{1}^{+}\left(t_{0}\right)=Y_{0} ; \\
\dot{Y}_{1}^{-}=-P_{1}^{-}(\dot{A}), & & Y_{1}^{-}\left(t_{0}\right)=Y_{1}^{+}\left(t_{1}\right) ; \\
& \vdots & & \\
\dot{Y}_{i}^{+} & =+P_{i}^{+}(\dot{A}), & & Y_{i}^{+}\left(t_{0}\right)=Y_{i-1}^{-}\left(t_{1}\right) ; \\
\dot{Y}_{i}^{-}=-P_{i}^{-}(\dot{A}), & Y_{i}^{-}\left(t_{0}\right)=Y_{i}^{+}\left(t_{1}\right) ; \\
\vdots & & \\
\dot{Y}_{d}^{+}=+P_{d}^{+}(\dot{A}), & & Y_{d}^{+}\left(t_{0}\right)=Y_{d-1}^{-}\left(t_{1}\right) .
\end{array}
$$


Here, $Y_{0}=Y\left(t_{0}\right)$ is the initial value of $(4.1)$ and $Y_{d}^{+}\left(t_{1}\right)$ is the final approximation for $Y\left(t_{1}\right)$. Observe that one full step consists of $2 d-1$ substeps.

We remark that the projectors $P_{i}^{+}, P_{i}^{-}$depend on the current value of $Y_{i}^{+}(t)$ or $Y_{i}^{-}(t)$; hence, they are in general time-dependent. For notational convenience, we do not denote this dependence explicitly since the following result states we can actually take them to be time-independent as long as they are updated after every substep. In addition, it shows how these substeps can be solved in closed form.

TheOREM 4.1. Let $\Delta A=A\left(t_{1}\right)-A\left(t_{0}\right)$. The initial value problems from above satisfy

$$
Y_{i}^{+}\left(t_{1}\right)=Y_{i}^{+}\left(t_{0}\right)+P_{i}^{+}(\Delta A) \quad \text { and } \quad Y_{i}^{-}\left(t_{1}\right)=Y_{i}^{-}\left(t_{0}\right)-P_{i}^{-}(\Delta A),
$$

where $P_{i}^{+}$and $P_{i}^{-}$are the projectors at $Y_{i}^{+}\left(t_{0}\right)$ and $Y_{i}^{-}\left(t_{0}\right)$, respectively.

In particular, if $Y_{i}^{+}\left(t_{0}\right)$ has the recursive $S V D$

$$
\left[Y_{i}^{+}\left(t_{0}\right)\right]^{\langle i\rangle}=\mathbf{Q}_{\leq i} \mathbf{S}_{i} \mathbf{Q}_{\geq i+1}^{\top}=\left(\mathbf{I}_{n_{i}} \otimes \mathbf{Q}_{\leq i-1}\right) \mathbf{Q}_{i}^{<} \mathbf{S}_{i} \mathbf{Q}_{\geq i+1}^{\top},
$$

with $\mathbf{Q}_{\leq 0}=\mathbf{Q}_{\geq d+1}=1$, then

$$
\left[Y_{i}^{+}\left(t_{1}\right)\right]^{\langle i\rangle}=\left(\mathbf{I}_{n_{i}} \otimes \mathbf{Q}_{\leq i-1}\right)\left\{\mathbf{Q}_{i}^{<} \mathbf{S}_{i}+\left(\mathbf{I}_{n_{i}} \otimes \mathbf{Q}_{\leq i-1}^{\top}\right)[\Delta A]^{\langle i\rangle} \mathbf{Q}_{\geq i+1}\right\} \mathbf{Q}_{\geq i+1}^{\top} .
$$

Likewise, if $Y_{i}^{-}\left(t_{0}\right)$ has the recursive $S V D$

$$
\left[Y_{i}^{-}\left(t_{0}\right)\right]^{\langle i\rangle}=\mathbf{Q}_{\leq i} \mathbf{S}_{i} \mathbf{Q}_{\geq i+1}^{\top},
$$

then

$$
\left[Y_{i}^{-}\left(t_{1}\right)\right]^{\langle i\rangle}=\mathbf{Q}_{\leq i}\left\{\mathbf{S}_{i}-\mathbf{Q}_{\leq i}^{\top}[\Delta A]^{\langle i\rangle} \mathbf{Q}_{\geq i+1}\right\} \mathbf{Q}_{\geq i+1}^{\top} .
$$

These results are furthermore valid for any ordering of the initial value problems.

Proof. First, observe that each $P_{i}^{+}$and $P_{i}^{-}$maps onto the current tangent space of $\mathcal{M}$ and that $Y_{0} \in \mathcal{M}$. Hence, each $Y_{i}^{+}(t)$ and $Y_{i}^{-}(t)$ will stay on $\mathcal{M}$. We may therefore assume that $Y_{i}^{+}(t)$ and $Y_{i}^{-}(t)$ admit TT/MPS decompositions of equal TT/MPS rank for $t \in\left[t_{0}, t_{1}\right]$.

By writing $Y_{i}^{+}(t)$ in a time-dependent recursive SVD,

$$
\left[Y_{i}^{+}(t)\right]^{\langle i\rangle}=\left(\mathbf{I} \otimes \mathbf{Q}_{\leq i-1}(t)\right) \mathbf{Q}_{i}^{<}(t) \mathbf{S}_{i}(t) \mathbf{Q}_{\geq i+1}^{\top}(t),
$$

we see from (4.2) that

$$
\left[P_{i}^{+}(\dot{A})\right]^{\langle i\rangle}=\left(\mathbf{I} \otimes \mathbf{Q}_{\leq i-1}(t) \mathbf{Q}_{\leq i-1}^{\top}(t)\right)[\dot{A}]^{\langle i\rangle} \mathbf{Q}_{\geq i+1}(t) \mathbf{Q}_{\geq i+1}^{\top}(t) .
$$

Hence the differential equation $\dot{Y}_{i}^{+}=P_{i}^{+}(\dot{A})$ implies

$$
\begin{aligned}
& \left(\mathbf{I} \otimes \dot{\mathbf{Q}}_{\leq i-1}(t)\right) \mathbf{Q}_{i}^{<}(t) \mathbf{S}_{i}(t) \mathbf{Q}_{\geq i+1}^{\top}(t)+\left(\mathbf{I} \otimes \mathbf{Q}_{\leq i-1}(t)\right) \frac{\mathrm{d}}{\mathrm{d} t}\left[\mathbf{Q}_{i}^{<}(t) \mathbf{S}_{i}(t)\right] \mathbf{Q}_{\geq i+1}^{\top}(t) \\
& \quad+\left(\mathbf{I} \otimes \mathbf{Q}_{\leq i-1}(t)\right) \mathbf{Q}_{i}^{<}(t) \mathbf{S}_{i}(t) \dot{\mathbf{Q}}_{\geq i+1}^{\top}(t) \\
& =\left(\mathbf{I} \otimes \mathbf{Q}_{\leq i-1}(t)\right)\left(\mathbf{I} \otimes \mathbf{Q}_{\leq i-1}^{\top}(t)\right)[\dot{A}]^{\langle i\rangle} \mathbf{Q}_{\geq i+1}(t) \mathbf{Q}_{\geq i+1}^{\top}(t) .
\end{aligned}
$$

By choosing $\dot{\mathbf{Q}}_{\leq i-1}(t)=0$ and $\dot{\mathbf{Q}}_{\geq i+1}(t)=0$, the above identity is satisfied when

$$
\frac{\mathrm{d}}{\mathrm{d} t}\left[\mathbf{Q}_{i}^{<}(t) \mathbf{S}_{i}(t)\right]=\left(\mathbf{I} \otimes \mathbf{Q}_{\leq i-1}^{\boldsymbol{T}}(t)\right)[\dot{A}]^{\langle i\rangle} \mathbf{Q}_{\geq i+1}(t) .
$$


Using the initial condition $Y_{i}^{+}(0)$, the solution of these differential equations becomes

$$
\begin{aligned}
& \mathbf{Q}_{\leq i-1}(t)=\mathbf{Q}_{\leq i-1}(0), \quad \mathbf{Q}_{\geq i+1}(t)=\mathbf{Q}_{\geq i+1}(0), \\
& \mathbf{Q}_{i}^{<}(t) \mathbf{S}_{i}(t)=\mathbf{Q}_{i}^{<}(0) \mathbf{S}_{i}(0)+\left(\mathbf{I} \otimes \mathbf{Q}_{\leq i-1}^{\boldsymbol{T}}(0)\right)[A(t)-A(0)]{ }^{\langle i\rangle} \mathbf{Q}_{\geq i+1}(0),
\end{aligned}
$$

which proves the statement for $\left[Y_{i}^{+}\left(t_{1}\right)\right]^{\langle i\rangle}$. Now, writing

$$
\begin{aligned}
{\left[Y_{i}^{+}\left(t_{1}\right)\right]^{\langle i\rangle} } & =\left(\mathbf{I} \otimes \mathbf{Q}_{\leq i-1}\right) \mathbf{Q}_{i}^{<} \mathbf{S}_{i} \mathbf{Q}_{\geq i+1}^{\top}+\left(\mathbf{I} \otimes \mathbf{Q}_{\leq i-1} \mathbf{Q}_{\leq i-1}^{\top}\right)[\Delta A]^{\langle i\rangle} \mathbf{Q}_{\geq i+1} \mathbf{Q}_{\geq i+1}^{\top} \\
& =\left[Y_{i}^{+}(0)\right]^{\langle i\rangle}+\left(\mathbf{I} \otimes \mathbf{P}_{\leq i-1}\right)[\Delta A]^{\langle i\rangle} \mathbf{P}_{\geq i+1},
\end{aligned}
$$

we have also proven the first statement of the theorem.

Since the previous derivation is valid for any initial condition, it does not depend on a specific ordering of the initial value problems. The derivation for $Y_{i}^{-}\left(t_{1}\right)$ is analogous to that of $Y_{i}^{+}\left(t_{1}\right)$. $\square$

In a similar way as for the proof of Corollary 3.2 , one can show that the projector (4.2) also satisfies

$$
P_{i}^{+}(Z)=\operatorname{Ten}_{i-1}\left[\mathbf{P}_{\leq i-1} \mathbf{Z}^{\langle i-1\rangle}\left(\mathbf{P}_{\geq i+1} \otimes \mathbf{I}_{n_{i}}\right)\right], \quad(1 \leq i \leq d) .
$$

This definition is useful when $Y_{i}^{+}\left(t_{0}\right)$ is given as (see $\S 4.3$ )

$$
\left[Y_{i}^{+}\left(t_{0}\right)\right]^{\langle i-1\rangle}=\mathbf{Q}_{\leq i-1} \mathbf{S}_{i-1} \mathbf{Q}_{i}^{>\boldsymbol{\top}}\left(\mathbf{Q}_{\geq i+1}^{\top} \otimes \mathbf{I}_{n_{i}}\right) .
$$

In that case, we have

$$
\left[Y_{i}^{+}\left(t_{1}\right)\right]^{\langle i-1\rangle}=\mathbf{Q}_{\leq i-1}\left\{\mathbf{S}_{i-1} \mathbf{Q}_{i}^{>\top}+\mathbf{Q}_{\leq i-1}^{\top}[\Delta A]^{\langle i-1\rangle}\left(\mathbf{Q}_{\geq i+1} \otimes \mathbf{I}_{n_{i}}\right)\right\}\left(\mathbf{Q}_{\geq i+1}^{\top} \otimes \mathbf{I}_{n_{i}}\right)
$$

4.2. Efficient implementation as a sweeping algorithm. Theorem 4.1 can be turned into an efficient scheme by updating the cores of the tensor $Y\left(t_{0}\right)$ from left to right. Our explanation will be high level, focusing only on pointing out which cores stay constant and which need to be updated throughout the sweep. A graphical depiction of the resulting procedure using tensor networks is given in Fig. 4.1. More detailed implementation issues are deferred to $§ 6.1$.

Preparation of $Y_{0}$. Before solving the substeps, we prepare the starting value $Y_{0}$ as follows. Write $Y=Y_{0}$ for notational convenience and suppose

$$
\mathbf{Y}^{\langle 1\rangle}\left(t_{0}\right)=\mathbf{Y}_{\leq 1}\left(t_{0}\right) \mathbf{Y}_{\geq 2}^{\boldsymbol{\top}}\left(t_{0}\right)
$$

By orthogonalization from the right we decompose $\mathbf{Y}_{\geq 2}\left(t_{0}\right)=\mathbf{Q}_{\geq 2}\left(t_{0}\right) \mathbf{R}_{2}\left(t_{0}\right)$, so that we obtain the right-orthogonalized factorization

$$
\mathbf{Y}^{\langle 1\rangle}\left(t_{0}\right)=\mathbf{K}_{1}^{<}\left(t_{0}\right) \mathbf{Q}_{\geq 2}^{\top}\left(t_{0}\right)
$$

with $\mathbf{K}_{1}^{<}\left(t_{0}\right)=\mathbf{Y}_{\leq 1}\left(t_{0}\right) \mathbf{R}_{2}^{\top}\left(t_{0}\right) \in \mathbb{R}^{n_{1} \times r_{1}}$ the first core of $Y\left(t_{0}\right)$.

Computation of $Y_{1}^{+}$. Denote $Y=Y_{1}^{+}$. Since $\mathbf{Q}_{\geq 2}^{\top}\left(t_{0}\right) \mathbf{Q}_{\geq 2}\left(t_{0}\right)=\mathbf{I}_{r_{1}}$, we have that $\mathbf{P}_{\geq 2}\left(t_{0}\right)=\mathbf{Q}_{\geq 2}\left(t_{0}\right) \mathbf{Q}_{\geq 2}^{\top}\left(t_{0}\right)$. Applying Theorem 4.1 gives

$$
\mathbf{Y}^{\langle 1\rangle}\left(t_{1}\right)=\mathbf{K}_{1}^{<}\left(t_{1}\right) \mathbf{Q}_{\geq 2}^{\top}\left(t_{0}\right),
$$

with

$$
\mathbf{K}_{1}^{<}\left(t_{1}\right)=\mathbf{K}_{1}^{<}\left(t_{0}\right)+\left(\mathbf{A}^{\langle 1\rangle}\left(t_{1}\right)-\mathbf{A}^{\langle 1\rangle}\left(t_{0}\right)\right) \mathbf{Q}_{\geq 2}\left(t_{0}\right) .
$$


Observe that compared to $Y\left(t_{0}\right)$ only the first core $K_{1}\left(t_{1}\right)$ of $Y\left(t_{1}\right)$ is changed, while all the others (that is, those that make up $\mathbf{Q}_{\geq 2}\left(t_{0}\right)$ ) stay constant. Hence, after computing the $\mathrm{QR}$ decomposition

$$
\mathbf{K}_{1}^{<}\left(t_{1}\right)=\mathbf{Q}_{1}^{<}\left(t_{1}\right) \mathbf{R}_{1}\left(t_{1}\right),
$$

we obtain a recursive SVD for $Y\left(t_{1}\right)=Y_{1}^{+}\left(t_{1}\right)$,

$$
\mathbf{Y}^{\langle 1\rangle}\left(t_{1}\right)=\mathbf{Q}_{\leq 1}\left(t_{1}\right) \mathbf{R}_{1}\left(t_{1}\right) \mathbf{Q}_{\geq 2}^{\top}\left(t_{0}\right) \quad \text { with } \quad \mathbf{Q}_{\leq 1}\left(t_{1}\right)=\mathbf{Q}_{1}^{<}\left(t_{1}\right)
$$

Computation of $Y_{i}^{-}$with $i=1, \ldots, d-1$. The computation for $Y_{1}^{-}$follows the same pattern as for arbitrary $Y_{i}^{-}$, so we explain it directly for $Y_{i}^{-}$.

We require that the initial value $Y_{i}^{-}\left(t_{0}\right)=Y_{i}^{+}\left(t_{1}\right)$ is available as a recursive SVD in node $i$. This is obviously true for $Y_{1}^{+}\left(t_{1}\right)$ and one can verify by induction that it is also true for $Y_{i}^{+}\left(t_{1}\right)$ with $i>1$, whose computation is explained below. Denoting $Y=Y_{i}^{-}$, we have in particular

$$
\mathbf{Y}^{\langle i\rangle}\left(t_{0}\right)=\mathbf{Q}_{\leq i}\left(t_{1}\right) \mathbf{R}_{i}\left(t_{1}\right) \mathbf{Q}_{\geq i+1}^{\top}\left(t_{0}\right)
$$

with $\mathbf{Q}_{\leq i}^{\top}\left(t_{1}\right) \mathbf{Q}_{\leq i}\left(t_{1}\right)=\mathbf{I}_{r_{i}}=\mathbf{Q}_{\geq i+1}^{\top}\left(t_{0}\right) \mathbf{Q}_{\geq i+1}\left(t_{0}\right)$. This means we can directly apply Theorem 4.1 for the computation of $Y\left(t_{1}\right)$ and obtain

$$
\mathbf{Y}^{\langle i\rangle}\left(t_{1}\right)=\mathbf{Q}_{\leq i}\left(t_{1}\right) \mathbf{S}_{i}\left(t_{1}\right) \mathbf{Q}_{\geq i+1}^{\top}\left(t_{0}\right)
$$

where $\mathbf{S}_{i}\left(t_{1}\right) \in \mathbb{R}^{r_{i} \times r_{i}}$ is given as

$$
\mathbf{S}_{i}\left(t_{1}\right)=\mathbf{R}_{i}\left(t_{1}\right)-\mathbf{Q}_{\leq i}^{\top}\left(t_{1}\right)\left(\mathbf{A}^{\langle i\rangle}(t)-\mathbf{A}^{\langle i\rangle}\left(t_{0}\right)\right) \mathbf{Q}_{\geq i+1}\left(t_{0}\right) .
$$

Observe that we maintain a recursive SVD in $i$ for $Y_{i}^{-}\left(t_{1}\right)$ without having to orthogonalize the matrices $\mathbf{Q}_{\leq i}\left(t_{1}\right)$ or $\mathbf{Q}_{\geq i+1}\left(t_{0}\right)$.

Computation of $Y_{i}^{+}$with $i=2, \ldots, d$. In this case, the initial value $Y_{i}^{+}\left(t_{0}\right)=$ $Y_{i-1}^{-}\left(t_{1}\right)$ is available as a recursive SVD in node $i-1$. Denoting $Y=Y_{i}^{+}$, then it is easily verified by induction that

$$
\mathbf{Y}^{\langle i-1\rangle}\left(t_{0}\right)=\mathbf{Q}_{\leq i-1}\left(t_{1}\right) \mathbf{S}_{i-1}\left(t_{1}\right) \mathbf{Q}_{\geq i}^{\top}\left(t_{0}\right)
$$

with $\mathbf{Q}_{\leq i-1}^{\boldsymbol{\top}}\left(t_{1}\right) \mathbf{Q}_{\leq i-1}\left(t_{1}\right)=\mathbf{I}_{r_{i-1}}=\mathbf{Q}_{\geq i}^{\boldsymbol{\top}}\left(t_{0}\right) \mathbf{Q}_{\geq i}\left(t_{0}\right)$. Recalling the relations (2.8) and (2.10), we can transform this $(i-1)$ th unfolding into the $i$ th unfolding,

$$
\mathbf{Y}^{\langle i\rangle}\left(t_{0}\right)=\left(\mathbf{I}_{n_{i}} \otimes \mathbf{Q}_{\leq i-1}\left(t_{1}\right)\right) \mathbf{K}_{i}^{<}\left(t_{0}\right) \mathbf{Q}_{\geq i+1}^{\top}\left(t_{0}\right)
$$

where $\mathbf{K}_{i}^{<}\left(t_{0}\right)=\left(\mathbf{I}_{n_{i}} \otimes \mathbf{S}_{i-1}\left(t_{1}\right)\right) \mathbf{Q}_{i}^{<}\left(t_{0}\right)$ is the left unfolding of the $i$ th core $K_{i}\left(t_{0}\right)$ of $Y\left(t_{0}\right)$. Theorem 4.1 then leads to

$$
\mathbf{Y}^{\langle i\rangle}\left(t_{1}\right)=\left(\mathbf{I}_{n_{i}} \otimes \mathbf{Q}_{\leq i-1}\left(t_{1}\right)\right) \mathbf{K}_{i}^{<}\left(t_{1}\right) \mathbf{Q}_{\geq i+1}^{\top}\left(t_{0}\right),
$$

where $\mathbf{K}_{i}^{<}\left(t_{1}\right) \in \mathbb{R}^{\left(r_{i-1} n_{i}\right) \times r_{i}}$ is given by

$$
\mathbf{K}_{i}^{<}\left(t_{1}\right)=\mathbf{K}_{i}^{<}\left(t_{0}\right)+\left(\mathbf{I}_{n_{i}} \otimes \mathbf{Q}_{\leq i-1}^{\boldsymbol{\top}}\left(t_{1}\right)\right)\left(\mathbf{A}^{\langle i\rangle}\left(t_{1}\right)-\mathbf{A}^{\langle i\rangle}\left(t_{0}\right)\right) \mathbf{Q}_{\geq i+1}\left(t_{0}\right) .
$$

Since now only the $i$ th core $K_{i}\left(t_{1}\right)$ of $Y\left(t_{1}\right)$ has changed, one QR decomposition

$$
\mathbf{K}_{i}^{<}\left(t_{1}\right)=\mathbf{Q}_{i}^{<}\left(t_{1}\right) \mathbf{R}_{i}\left(t_{1}\right)
$$


0) Prepared for $Y_{i}^{+}$

$$
Q_{\leq i-1}\left(t_{1}\right) \quad K_{i}\left(t_{0}\right) \quad Q_{\geq i+1}\left(t_{0}\right) \quad \text { 1) Compute contraction }
$$

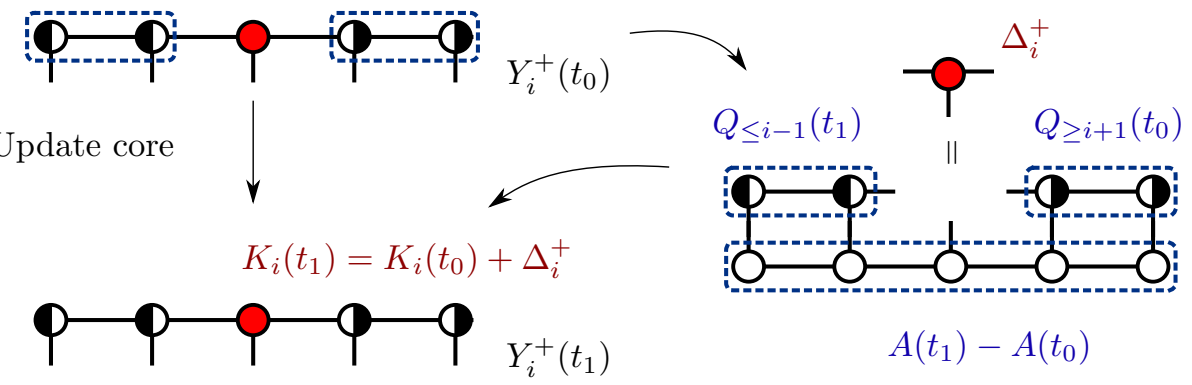

3) Orthogonalize ॥

$Q_{\leq i}\left(t_{1}\right) \quad Q_{i}\left(t_{1}\right) \quad R_{i}\left(t_{1}\right)$

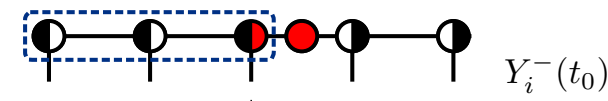

5) Update core

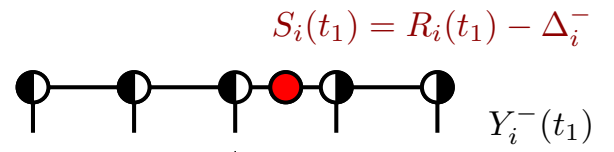

6) Prepare for $Y_{i+1}^{+} \downarrow$

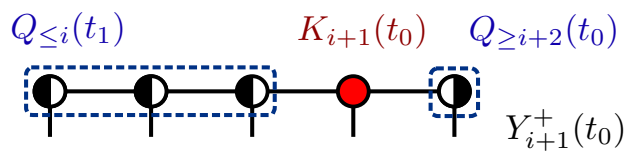

4) Compute contraction

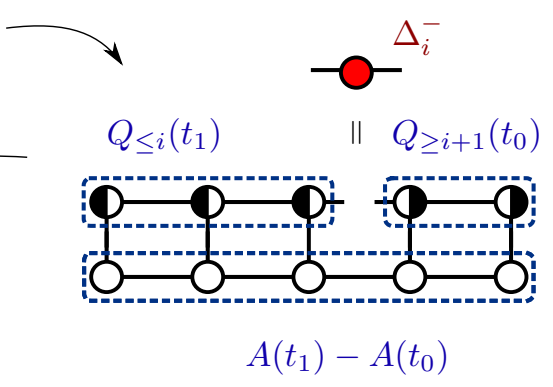

FIG. 4.1. The two sweeping algorithms update the cores selectively throughout the time stepping computations. Shown for the forward sweep when computing $Y_{i}^{+}$and $Y_{i}^{-}$.

suffices to obtain a recursive SVD of $Y_{i}^{+}\left(t_{1}\right)=Y\left(t_{1}\right)$ at node $i$,

$\mathbf{Y}^{\langle i\rangle}\left(t_{1}\right)=\mathbf{Q}_{\leq i}\left(t_{1}\right) \mathbf{R}_{i}\left(t_{1}\right) \mathbf{Q}_{\geq i+1}^{\boldsymbol{\top}}\left(t_{0}\right), \quad$ with $\quad \mathbf{Q}_{\leq i}\left(t_{1}\right)=\left(\mathbf{I}_{n_{i}} \otimes \mathbf{Q}_{\leq i-1}\left(t_{1}\right)\right) \mathbf{Q}_{i}^{<}\left(t_{1}\right)$

Next time step. The final step $Y_{d}^{+}\left(t_{1}\right)$ will be an approximation to $Y\left(t_{1}\right)$ and consists of a left-orthogonal $\mathbf{Q}_{<d}\left(t_{1}\right)$. If we now want to continue with the time stepper to approximate $Y\left(t_{2}\right)$ for $t_{2}>t_{1}$, we need to apply the scheme again using $Y_{d}^{+}\left(t_{1}\right)$ as initial value. This requires a new orthogonalization procedure from right to left, since the initial value for the sweep has to be right orthogonalized.

4.3. Second-order scheme by a back-and-forth sweep. In many cases, it is advisable to compose the scheme from above with its adjoint instead of only orthogo- 
nalizing and continuing with the next step. In particular, the Strang splitting consists of first computing the original splitting scheme on $t \in\left[t_{0}, t_{1 / 2}\right]$ with $t_{1 / 2}=\left(t_{1}+t_{0}\right) / 2$ and then applying the adjoint of this scheme on $t \in\left[t_{1 / 2}, t_{1}\right]$. The result will be a symmetric time stepper of order two; see, e.g., [8, II.5].

For our splitting, the adjoint step is simply solving the split differential equations in reverse order. Since Theorem 4.1 is independent of the ordering of the differential equations, we can again use its closed-form solutions to derive an efficient sweeping algorithm for this adjoint step. We briefly explain the first three steps and refer to Algorithm 1 for the full second-order scheme. Observe that this scheme can be seen as a full back-and-forth sweep.

Denote the final step of the forward sweep on $t \in\left[t_{0}, t_{1 / 2}\right]$ by $\widehat{Y}=Y_{d}^{+}\left(t_{1 / 2}\right)$. It satisfies (recall that $t_{1}$ takes the role of $t_{1 / 2}$ in the derivations above)

$$
\widehat{\mathbf{Y}}^{\langle d\rangle}\left(t_{1 / 2}\right)=\left(\mathbf{I}_{n_{d}} \otimes \mathbf{Q}_{\leq d-1}\left(t_{1 / 2}\right)\right) \mathbf{K}_{d}^{<}\left(t_{1 / 2}\right) .
$$

with

$$
\mathbf{K}_{d}^{<}\left(t_{1 / 2}\right)=\mathbf{K}_{d}^{<}\left(t_{0}\right)+\left(\mathbf{I}_{n_{d}} \otimes \mathbf{Q}_{\leq d-1}^{\boldsymbol{\top}}\left(t_{1 / 2}\right)\right)\left(\mathbf{A}^{\langle d\rangle}\left(t_{1 / 2}\right)-\mathbf{A}^{\langle d\rangle}\left(t_{0}\right)\right) .
$$

The first substep of the adjoint scheme consists of solving

$$
\dot{Y}_{d}^{+}=P_{d}^{+}(\dot{A}), \quad Y_{d}^{+}\left(t_{1 / 2}\right)=\widehat{Y},
$$

for $t \in\left[t_{1 / 2}, t_{1}\right]$. Denote $Y=Y_{d}^{+}$. We can directly apply Theorem 4.1 to obtain

$$
\mathbf{Y}^{\langle d\rangle}\left(t_{1}\right)=\left(\mathbf{I}_{n_{d}} \otimes \mathbf{Q}_{\leq d-1}\left(t_{1 / 2}\right)\right) \mathbf{K}_{d}^{<}\left(t_{1}\right)
$$

with

$$
\begin{aligned}
\mathbf{K}_{d}^{<}\left(t_{1}\right) & =\mathbf{K}_{d}^{<}\left(t_{1 / 2}\right)+\left(\mathbf{I}_{n_{d}} \otimes \mathbf{Q}_{\leq d-1}^{\top}\left(t_{1 / 2}\right)\right)\left(\mathbf{A}^{\langle d\rangle}\left(t_{1}\right)-\mathbf{A}^{\langle d\rangle}\left(t_{1 / 2}\right)\right) \\
& =\mathbf{K}_{d}^{<}\left(t_{0}\right)+\left(\mathbf{I}_{n_{d}} \otimes \mathbf{Q}_{\leq d-1}^{\top}\left(t_{1 / 2}\right)\right)\left(\mathbf{A}^{\langle d\rangle}\left(t_{1}\right)-\mathbf{A}^{\langle d\rangle}\left(t_{0}\right)\right) .
\end{aligned}
$$

Hence, the last substep of the forward sweep and the first of the backward sweep can be combined into one.

The second substep amounts to solving

$$
\dot{Y}_{d-1}^{-}=-P_{d-1}^{-}(\dot{A}), \quad Y_{d-1}^{-}\left(t_{1 / 2}\right)=Y_{d}^{+}\left(t_{1}\right) .
$$

Let $Y=Y_{d-1}^{-}$. Then we can write the initial condition as

$$
\mathbf{Y}^{\langle d-1\rangle}\left(t_{1 / 2}\right)=\mathbf{Q}_{\leq d-1}\left(t_{1 / 2}\right) \mathbf{K}_{d}^{>}\left(t_{1}\right)^{\top}
$$

and using the QR decomposition $\mathbf{K}_{d}^{>}\left(t_{1}\right)=\mathbf{Q}_{d}^{>}\left(t_{1}\right) \mathbf{R}_{d-1}\left(t_{1}\right)$ also as

$$
\mathbf{Y}^{\langle d-1\rangle}\left(t_{1 / 2}\right)=\mathbf{Q}_{\leq d-1}\left(t_{1 / 2}\right) \mathbf{R}_{d-1}^{\top}\left(t_{1}\right) \mathbf{Q}_{\geq d}^{\top}\left(t_{1}\right),
$$

where $\mathbf{Q}_{\geq d}\left(t_{1}\right)=\mathbf{Q}_{d}^{>}\left(t_{1}\right)$. Applying Theorem 4.1, we obtain

$$
\mathbf{Y}^{\langle d-1\rangle}\left(t_{1}\right)=\mathbf{Q}_{\leq d-1}\left(t_{1 / 2}\right) \mathbf{S}_{d-1}^{\boldsymbol{\top}}\left(t_{1}\right) \mathbf{Q}_{\geq d}^{\top}\left(t_{1}\right),
$$

where

$$
\mathbf{S}_{d-1}^{\boldsymbol{\top}}\left(t_{1}\right)=\mathbf{R}_{d-1}^{\boldsymbol{\top}}\left(t_{1}\right)-\mathbf{Q}_{\leq d-1}^{\boldsymbol{\top}}\left(t_{1 / 2}\right)\left(\mathbf{A}^{\langle d-1\rangle}\left(t_{1}\right)-\mathbf{A}^{\langle d-1\rangle}\left(t_{1 / 2}\right)\right) \mathbf{Q}_{\geq d}\left(t_{1}\right) .
$$


For the third substep

$$
\dot{Y}_{d-1}^{+}=P_{d-1}^{-}(\dot{A}), \quad Y_{d-1}^{+}\left(t_{1 / 2}\right)=Y_{d-1}^{-}\left(t_{1}\right),
$$

we denote $Y=Y_{d-1}^{+}$. In this case, unfold using (2.12) and (2.13) the computed quantity $Y_{d-1}\left(t_{1}\right)$ from above as

$$
\mathbf{Y}^{\langle d-2\rangle}\left(t_{1}\right)=\mathbf{Q}_{\leq d-2}\left(t_{1 / 2}\right) \mathbf{K}_{d-1}^{>\top}\left(t_{1 / 2}\right)\left(\mathbf{Q}_{\geq d}^{\top}\left(t_{1}\right) \otimes \mathbf{I}_{n_{d-1}}\right)
$$

with $\mathbf{K}_{d-1}^{>\boldsymbol{\top}}\left(t_{1 / 2}\right)=\mathbf{Q}_{d-1}^{>\boldsymbol{\top}}\left(t_{1 / 2}\right)\left(\mathbf{S}_{d-1}^{\boldsymbol{\top}}\left(t_{1}\right) \otimes \mathbf{I}_{n_{d-1}}\right)$. From here on, all subsequent computations are straightforward if we use (4.6) to compute $Y_{i}^{+}\left(t_{1}\right)$.

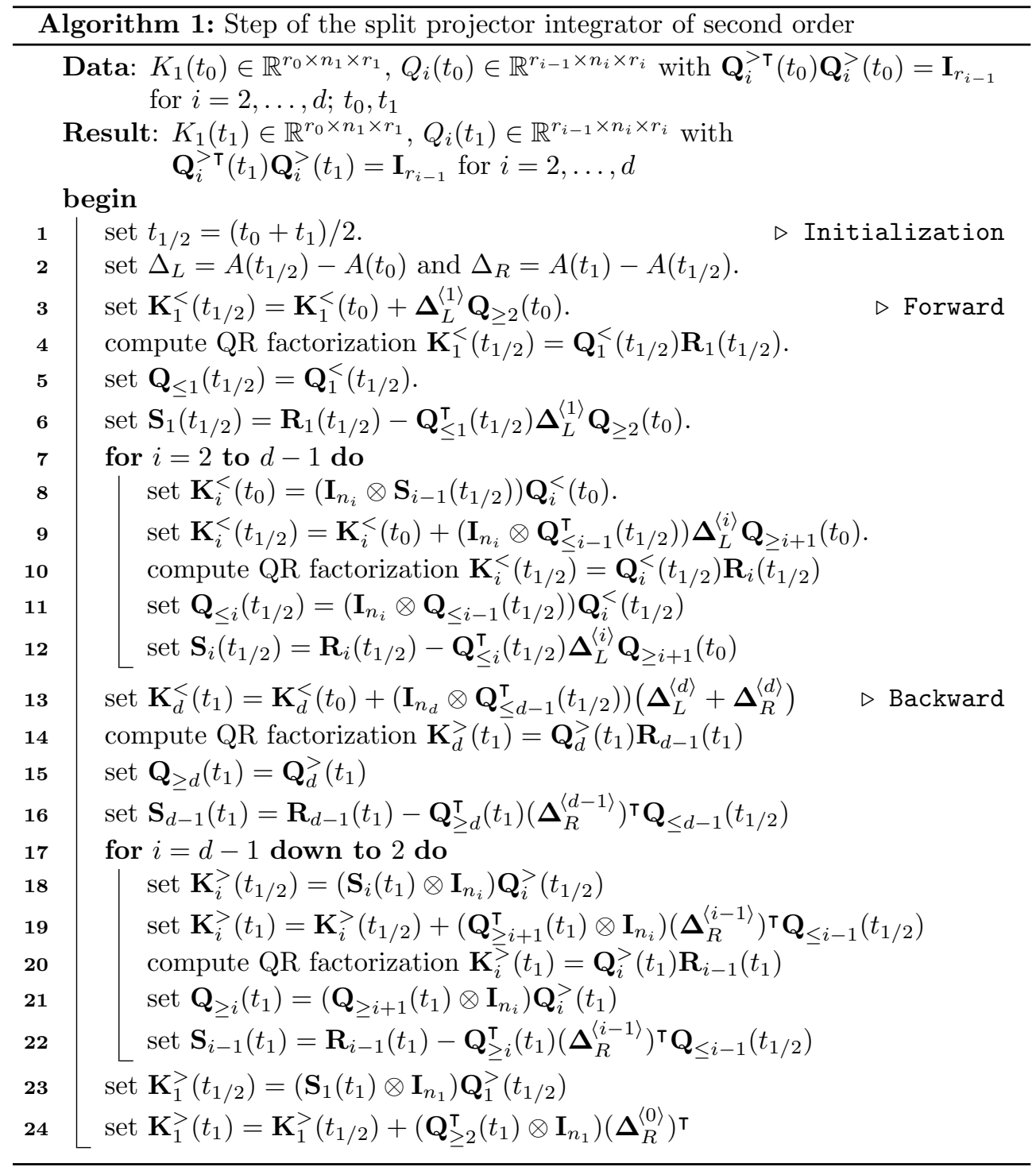


5. Exactness property of the integrator. We show that the splitting integrator is exact when $A(t)$ is a tensor of constant TT/MPS rank $\mathbf{r}$. This is similar to Theorem 4.1 in [19] for the matrix case, except that in our case we require the rank of $A(t)$ to be exactly $\mathbf{r}$ and not merely bounded by $\mathbf{r}$. Note, however, that the positive singular values of unfoldings of $A(t)$ can be arbitrarily small.

Theorem 5.1. Suppose $A(t) \in \mathcal{M}$ for $t \in\left[t_{0}, t_{1}\right]$. Then, for sufficiently small $t_{1}-t_{0}>0$ the splitting integrators of orders one and two are exact when started from $Y_{0}=A\left(t_{0}\right)$. For example, $Y_{d}^{+}\left(t_{1}\right)=A\left(t_{1}\right)$ for the first-order integrator.

The proof of this theorem follows trivially from the following lemma.

Lemma 5.2. Suppose $A(t) \in \mathcal{M}$ for $t \in\left[t_{0}, t_{1}\right]$ with recursive $S V D s$

$$
[\mathbf{A}(t)]^{\langle i\rangle}=\mathbf{Q}_{\leq i}(t) \mathbf{S}_{i}(t) \mathbf{Q}_{\geq i+1}^{\top}(t) \quad \text { for } i=0,1, \ldots, d .
$$

Let $Y_{0}=A\left(t_{0}\right)$, then for sufficiently small $t_{1}-t_{0}>0$ the consecutive steps in the splitting integrator of $\S 4.1$ satisfy

$$
Y_{i}^{+}\left(t_{1}\right)=P_{\geq i+1}^{(0)} A\left(t_{1}\right) \quad \text { and } \quad Y_{i}^{-}\left(t_{1}\right)=P_{\leq i}^{(1)} A\left(t_{0}\right) \quad \text { for } i=1,2, \ldots, d,
$$

where

$$
\begin{aligned}
& P_{\geq i+1}^{(0)} Z=\operatorname{Ten}_{i}\left(\mathbf{Z}^{\langle i\rangle} \mathbf{Q}_{\geq i+1}\left(t_{0}\right) \mathbf{Q}_{\geq i+1}^{\top}\left(t_{0}\right)\right) \\
& P_{\leq i}^{(1)} Z=\operatorname{Ten}_{i}\left(\mathbf{Q}_{\leq i}\left(t_{1}\right) \mathbf{Q}_{\leq i}^{\top}\left(t_{1}\right) \mathbf{Z}^{\langle i\rangle}\right) .
\end{aligned}
$$

Before proving this lemma, we point out that the assumption of sufficiently small $t_{1}-t_{0}$ is only because the matrices $\mathbf{Q}_{\leq i-1}^{\boldsymbol{\top}}\left(t_{1}\right) \mathbf{Q}_{\leq i-1}\left(t_{0}\right)$ and $\mathbf{Q}_{\geq i}^{\boldsymbol{\top}}\left(t_{1}\right) \mathbf{Q}_{\geq i}\left(t_{0}\right)$ need to be invertible. Since the full column-rank matrices $\mathbf{Q}_{\leq i}(t)$ and $\mathbf{Q}_{\geq i}(t)$ can be chosen continuous functions in $t$, this is always satisfied for $t_{1}-t_{0}$ sufficiently small. It may however also hold for larger values of $t_{1}-t_{0}$.

Proof. The proof proceeds by induction on $i$ from left to right. Since $Y_{1}^{+}\left(t_{0}\right)=$ $A\left(t_{0}\right)$, we can include the case for $Y_{1}^{+}\left(t_{1}\right)$ in our proof below for general $i$ by putting $Y_{0}^{-}\left(t_{1}\right)=Y_{0}^{+}\left(t_{0}\right)$ and $P_{\leq 0}^{(1)}=1$.

Now, suppose the statement to be true for $i>1$. Then, $Y_{i}^{+}\left(t_{0}\right)=Y_{i-1}^{-}\left(t_{1}\right)=$ $P_{\leq i-1}^{(1)} A\left(t_{0}\right)$, which gives

$$
\begin{aligned}
{\left[\mathbf{Y}_{i}^{+}\left(t_{0}\right)\right]^{\langle i-1\rangle} } & =\mathbf{Q}_{\leq i-1}\left(t_{1}\right) \mathbf{Q}_{\leq i-1}^{\top}\left(t_{1}\right) \mathbf{Q}_{\leq i-1}\left(t_{0}\right) \mathbf{S}_{i-1}\left(t_{0}\right) \mathbf{Q}_{\geq i}^{\top}\left(t_{0}\right) \\
& =\mathbf{Q}_{\leq i-1}\left(t_{1}\right) \mathbf{S}_{i-1}^{+} \mathbf{Q}_{\geq i}^{\top}\left(t_{0}\right) .
\end{aligned}
$$

Observe that $Y_{i}^{+}\left(t_{0}\right) \in \mathcal{M}$ since $\mathbf{S}_{i-1}^{+}=\mathbf{Q}_{\leq i-1}^{\top}\left(t_{1}\right) \mathbf{Q}_{\leq i-1}\left(t_{0}\right) \mathbf{S}_{i-1}\left(t_{0}\right)$ is of full rank for $t_{1}-t_{0}$ sufficiently small. Hence, from (2.8)-(2.10) we obtain

$$
\left[\mathbf{Y}_{i}^{+}\left(t_{0}\right)\right]^{\langle i\rangle}=\left(\mathbf{I}_{n_{i}} \otimes \mathbf{Q}_{\leq i-1}\left(t_{1}\right)\right)\left(\mathbf{I}_{n_{i}} \otimes \mathbf{S}_{i-1}^{+}\right) \mathbf{Q}_{i}^{<\boldsymbol{\top}}\left(t_{0}\right) \mathbf{Q}_{\geq i+1}^{\top}\left(t_{0}\right) .
$$

Comparing to (4.2), we see that the projector onto the tangent space at $Y_{i}^{+}\left(t_{0}\right)$ equals $P_{i}^{+}=P_{\leq i-1}^{(1)} P_{\geq i+1}^{(0)}=P_{\geq i+1}^{(0)} P_{\leq i-1}^{(1)}$. The previous identities give with Theorem 4.1 that

$$
\begin{aligned}
Y_{i}^{+}\left(t_{1}\right) & =Y_{i}^{+}\left(t_{0}\right)+P_{i}^{+} A\left(t_{1}\right)-P_{i}^{+} A\left(t_{0}\right) \\
& =P_{\leq i-1}^{(1)} A\left(t_{0}\right)+P_{\geq i+1}^{(0)} P_{\leq i-1}^{(1)} A\left(t_{1}\right)-P_{\leq i-1}^{(1)} P_{\geq i+1}^{(0)} A\left(t_{0}\right)=P_{\geq i+1}^{(0)} A\left(t_{1}\right),
\end{aligned}
$$


where we used $P_{\geq i+1}^{(0)} A\left(t_{0}\right)=A\left(t_{0}\right)$ and $P_{\leq i-1}^{(1)} A\left(t_{1}\right)=A\left(t_{1}\right)$.

Continuing with $Y_{i}^{-}\left(t_{0}\right)=Y_{i}^{+}\left(t_{1}\right)=P_{\geq i+1}^{(0)} A\left(t_{1}\right)$, we have

$$
\begin{aligned}
{\left[Y_{i}^{-}\left(t_{0}\right)\right]^{\langle i\rangle} } & =\mathbf{Q}_{\leq i}\left(t_{1}\right) \mathbf{S}_{i}\left(t_{1}\right) \mathbf{Q}_{\geq i+1}^{\boldsymbol{\top}}\left(t_{1}\right) \mathbf{Q}_{\geq i+1}\left(t_{0}\right) \mathbf{Q}_{\geq i+1}^{\top}\left(t_{0}\right) \\
& =\mathbf{Q}_{\leq i}\left(t_{1}\right) \mathbf{S}_{i}^{-} \mathbf{Q}_{\geq i+1}^{\boldsymbol{\top}}\left(t_{0}\right) .
\end{aligned}
$$

This is again a recursive SVD with full rank $\mathbf{S}_{i}^{-}=\mathbf{S}_{i}\left(t_{1}\right) \mathbf{Q}_{\geq i+1}^{\top}\left(t_{1}\right) \mathbf{Q}_{\geq i+1}\left(t_{0}\right)$. Comparing to (4.3), we have $P_{i}^{-}=P_{\leq i}^{(1)} P_{\geq i+1}^{(0)}=P_{\geq i+1}^{(0)} P_{\leq i}^{(1)}$ and Theorem 4.1 gives

$$
\begin{aligned}
Y_{i}^{-}\left(t_{1}\right) & =Y_{i}^{-}\left(t_{0}\right)-P_{i}^{-} A\left(t_{1}\right)+P_{i}^{-} A\left(t_{0}\right) \\
& =P_{\geq i+1}^{(0)} A\left(t_{1}\right)-P_{\geq i+1}^{(0)} P_{\leq i}^{(1)} A\left(t_{1}\right)+P_{\leq i}^{(1)} P_{\geq i+1}^{(0)} A\left(t_{0}\right)=P_{\leq i}^{(1)} A\left(t_{0}\right),
\end{aligned}
$$

where we used $P_{\leq i}^{(1)} A\left(t_{1}\right)=A\left(t_{1}\right)$. This concludes the proof.

Now, Theorem 5.1 is a simple corollary.

Proof of Theorem 5.1. For the forward sweep (that is, the first-order scheme), Lemma 5.2 immediately gives exactness since $Y_{d}^{+}\left(t_{1}\right)=P_{\geq d+1}^{(0)} A\left(t_{1}\right)=A\left(t_{1}\right)$ with $\mathbf{Q}_{\geq d+1}\left(t_{0}\right)=1$. The second-order scheme composes this forward sweep with a backward sweep involving the same substeps. It is not difficult to prove the analogous version of Lemma 5.2 for such a backward ordering such that we establish exactness for the second-order scheme too.

6. Numerical implementation and experiments. We consider two numerical experiments. First, we use the splitting integrator for the integration of a timedependent molecular Schrödinger equation with a model potential. In the second experiment, we use one step of the splitting integrator as a retraction on the manifold of TT/MPS tensors and perform a Newton-Schultz iteration for approximate matrix inversion.

6.1. Implementation details. As explained in $\S 4.2-4.3$, the integrator updates the cores $K_{i}$ and matrices $\mathbf{S}_{i}$ in a forward, and possibly, backward ordering. Except for the (relatively cheap) orthogonalizations of the cores, the most computationally intensive part of the algorithm is computing these updates. For example, in the forward sweep, we need to compute the contractions (see Fig. 4.1)

$$
\begin{aligned}
\Delta_{i}^{+} & =\left(\mathbf{I} \otimes \mathbf{Q}_{\leq i-1}^{\boldsymbol{\top}}\left(t_{1}\right)\right)\left[A\left(t_{1}\right)-A\left(t_{0}\right)\right]^{\langle i\rangle} \mathbf{Q}_{\geq i+1}\left(t_{0}\right), \\
\Delta_{i}^{-} & =\mathbf{Q}_{\leq i}^{\boldsymbol{\top}}\left(t_{1}\right)\left[A\left(t_{1}\right)-A\left(t_{0}\right)\right]^{\langle i\rangle} \mathbf{Q}_{\geq i+1}\left(t_{0}\right) .
\end{aligned}
$$

It is highly recommended to avoid constructing the matrices $\mathbf{Q}_{\leq i}$ and $\mathbf{Q}_{\geq i}$ explicitly when computing $\Delta_{i}^{+}, \Delta_{i}^{-}$and instead exploit their TT/MPS structure. How this can be done, depends mostly on the structure of the increments $A\left(t_{1}\right)-A\left(t_{0}\right)$. In particular, the contractions are computed inexpensively if $A(t)$ is itself a linear combination of TT/MPS tensors, possibly having different rank than $Y$, and a sparse tensor.

The computation of $K_{i}$ and $\mathbf{S}_{i}$ changes when the tensor $A(t)$ is not given explicitly, but determined as the solution of a tensor differential equation

$$
\dot{A}(t)=F(t, A(t)) .
$$

In case of a forward sweep, $Y_{i}^{+}\left(t_{1}\right)$ is obtained as the evaluation at $t=t_{1}$ of

$$
Y_{i}^{+}(t)=\left(\mathbf{I} \otimes \mathbf{Q}_{\leq i-1}\left(t_{1}\right)\right) \mathbf{K}_{i}^{<}(t) \mathbf{Q}_{\geq i+1}^{\boldsymbol{\top}}\left(t_{0}\right),
$$


where $\mathbf{K}_{i}^{<}(t)=\mathbf{Q}_{i}^{<}(t) \mathbf{S}_{i}(t)$ satisfies (4.4). Hence, for $\dot{A}(t)=F(t, Y(t))$, we obtain

$$
\dot{\mathbf{K}}_{i}^{<}=\left(\mathbf{I} \otimes \mathbf{Q}_{\leq i-1}^{\top}\left(t_{1}\right)\right)\left[F\left(t, Y_{i}^{+}(t)\right)\right]^{\langle i\rangle} \mathbf{Q}_{\geq i+1}\left(t_{0}\right) .
$$

In an analogous way, the result of the next substep $Y_{i}^{-}\left(t_{1}\right)$ is obtained from

$$
\begin{aligned}
& Y_{i}^{-}(t)=\mathbf{Q}_{\leq i}\left(t_{1}\right) \mathbf{S}_{i}(t) \mathbf{Q}_{\geq i+1}^{\top}\left(t_{0}\right), \\
& \left.\dot{\mathbf{S}}_{i}=-\mathbf{Q}_{\leq i}^{\boldsymbol{\top}}\left(t_{1}\right)\right)\left[F\left(t, Y_{i}^{-}(t)\right)\right]^{\langle i\rangle} \mathbf{Q}_{\geq i+1}\left(t_{0}\right) .
\end{aligned}
$$

These differential equations can be solved numerically by a Runge-Kutta method (of order at least 2 for the second-order splitting integrator). In the important particular case of an autonomous linear ODE

$$
\dot{A}(t)=F(t, A(t))=L(A(t)), \quad \text { with linear } L: \mathbb{R}^{n_{1} \times \cdots \times n_{d}} \rightarrow \mathbb{R}^{n_{1} \times \cdots \times n_{d}},
$$

the above differential equations are constant-coefficient linear differential equations for $\mathbf{K}_{i}^{<}$and $\mathbf{S}_{i}$, respectively, which can be solved efficiently with a few iterations of a Krylov subspace method for computing the action of the operator exponential [9, 30, 10].

6.2. Quantum dynamics in a model potential. Quantum molecular dynamics is one of the promising applications of the split projector integrator. As a test problem, we use the same setup as considered in [24]: the time-dependent Schrödinger equation with Henon-Heiles potential modeling a coupled oscillator,

$$
i \frac{d \psi}{d t}=H \psi, \quad \psi(0)=\psi_{0},
$$

where the Hamiltonian operator $H$ has the form

$$
H\left(q_{1}, \ldots, q_{f}\right)=\overbrace{-\frac{1}{2} \Delta+\underbrace{\frac{1}{2} \sum_{k=1}^{f} q_{k}^{2}}_{\text {Henon-Heiles potential } V\left(q_{1}, \ldots, q_{f}\right)}+\overbrace{\lambda \sum_{k=1}^{f-1}\left(q_{k}^{2} q_{k+1}-\frac{1}{3} q_{k+1}^{3}\right)}^{\text {harmonic part }}}^{\text {anharmonic part }}
$$

with $\lambda=0.111803$. As an initial condition $\psi_{0}$, we choose a product of shifted Gaussians,

$$
\psi_{0}=\prod_{i=1}^{f} \exp \left(-\frac{(q-2)^{2}}{2}\right) .
$$

The correct discretization of such problems is delicate. A standard approach is to use a Discrete Variable Representation (DVR), specifically, the Sine-DVR scheme from [3]. In addition, since the problem is defined over the whole space, appropriate boundary conditions are required. We use complex absorbing potentials (CAP) of the form (see, for example, [22])

$$
W(q)=i \eta \sum_{i=1}^{f}\left(\left(q_{i}-q_{i}^{(r)}\right)_{+}^{b_{r}}+\left(q_{i}-q_{i}^{(l)}\right)_{-}^{b_{l}}\right),
$$


where

$$
z_{+}=\left\{\begin{array}{ll}
z, & \text { if } z \geq 0, \\
0, & \text { otherwise }
\end{array} \quad \text { and } \quad z_{-}= \begin{cases}z, & \text { if } z \leq 0 \\
0, & \text { otherwise }\end{cases}\right.
$$

The parameters $q_{i}^{(r)}$ and $q_{i}^{(l)}$ specify the effective boundary of the domain. CAP reduces the reflection from the boundary back to the domain, but the system is no longer conservative. For the Henon-Heiles example from above we have chosen

$$
\eta=-1, \quad q_{i}^{(l)}=-6, \quad q_{i}^{(r)}=6, \quad b_{r}=b_{l}=3 .
$$

We compute the dynamics using the second-order splitting integrator where the (linear) local problems for $K_{i}, \mathbf{S}_{i}$ are integrated using the Expokit package [30] with a relative accuracy of $10^{-8}$.

In order to evaluate the accuracy and efficiency of our proposed splitting integrator, we performed a preliminary comparison with the multi-configuration timedependent Hartree (MCTDH) package [32]. The MCTDH method [22] is the de-facto standard for doing high-dimensional quantum molecular dynamics simulations. For the detailed description of MCTDH, we refer to [22, 23, 2, 21].

As numerical experiment, we run MCTDH for the 10-dimensional Henon-Heiles problem from above with mode-folding. This can be considered as a first step of the hierarchical Tucker format (in this context called the multilayer MCTDH decomposition) with 32 basis functions in each mode, and the resulting function was approximated by a 5 -dimensional tensor with mode sizes equal to 18 . The final time was $T=60$. Our splitting integrator solved the same Henon-Heiles problem but now using the second-order splitting integrator with a fixed time step $h=0.01$. Except that we use the TT/MPS manifold for our scheme instead of a Tucker-type manifold as in MCDTH, all other computational parameters are the same.

In Fig. 6.1 we see the vibrational spectrum of a molecule, which is obtained as follows. After the dynamical low-rank approximation $\psi(t)$ is computed, we evaluate the autocorrelation function $a(t)=\langle\psi(t), \psi(0)\rangle$, and compute its Fourier transform $\widehat{a}(\xi)$. The absolute value of $\widehat{a}(\xi)$ gives the information about the energy spectrum of the operator. If the dynamics is approximated sufficiently accurately, the function $\widehat{a}(\xi)$ is approximated as a sum of delta functions located at the eigenvalues of $H$. This method can be considered as a method to approximate many eigenvalues of $H$ by using only one solution of the dynamical problem, which is not typical to standard numerical analysis, but often used in chemistry.

We see in Fig. 6.1 that the computed spectra are very similar, but the MCTDH computation took 54354 seconds, whereas the splitting integrator scheme took only 4425 seconds. A detailed comparison of the splitting scheme and MCTDH for quantum molecular dynamics will be presented elsewhere. This will include different benchmark problems and a comparison with the multilayer version of the MCTDH. 


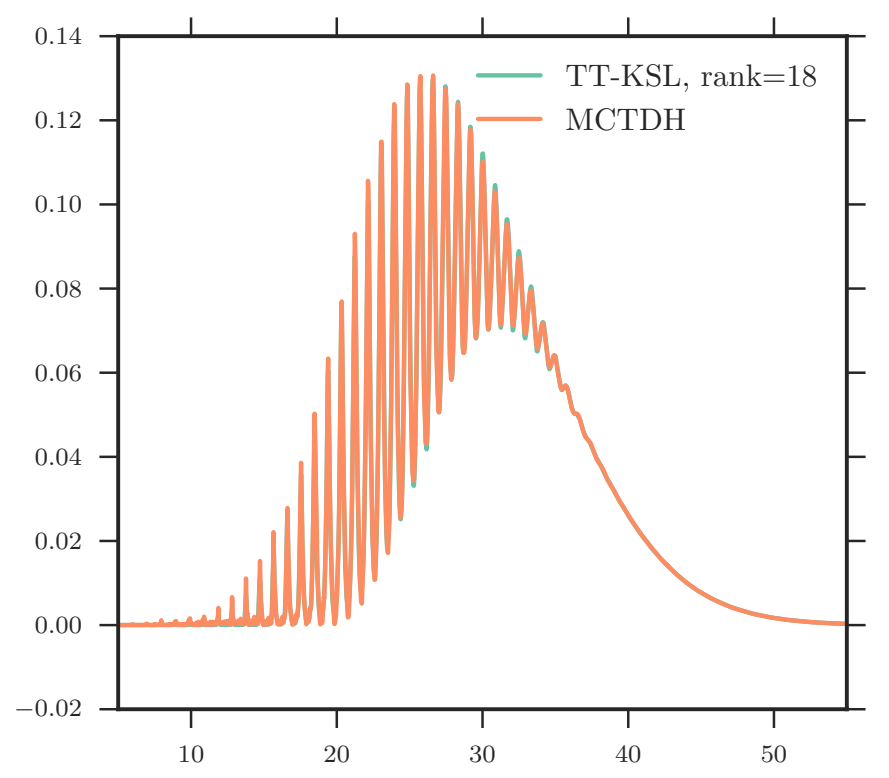

FIG. 6.1. Spectrum computed by the second-order splitting integrator and by the MCTDH package

6.3. Approximate matrix inversion. Optimization on low-rank tensor manifolds is another promising application of the splitting integrator scheme and can be rather easily incorporated. Consider some iterative process of the form

$$
Y_{k+1}=Y_{k}+\Delta_{k} \quad k=0, \ldots
$$

where $\Delta_{k}$ is the update. In order to obtain approximations $Z_{k} \in \mathcal{M}$ of $Y_{k}$ in the TT/MPS format, one typically retracts the new iterate back to $\mathcal{M}$,

$$
Z_{k+1}=P_{r}\left(Z_{k}+\Delta_{k}\right)
$$

with $P_{r}: \mathbb{R}^{n_{1} \times \cdots \times n_{d}} \rightarrow \mathcal{M}$ a retraction; see [1]. A widely used choice for $P_{r}$ is the quasi-optimal projection computed by TT-SVD [26]. Instead, we propose the cheaper alternative of one step of Algorithm 1 with $A\left(t_{1}\right)-A\left(t_{0}\right)=\Delta_{k}$ as $P_{r}$. In practice, the intermediate quantities in Algorithm 1 have to be computed without forming $\Delta_{k}$ explicitly. This can be done, for example, when $\Delta_{k}$ is a TT/MPS tensor of low-rank as explained in $\S 6.1$.

An important example of (6.3) is the Newton-Schultz iteration for the approximate matrix inversion (see, e.g., [6]),

$$
Y_{k+1}=2 Y_{k}-Y_{k} A Y_{k}, \quad k=0, \ldots
$$

It is well-known that iteration (6.4) converges quadratically provided that $\rho\left(I-A Y_{0}\right) \leq$ 1 , where $\rho(\cdot)$ is the spectral radius of the matrix. The matrix $A$ is supposed to have low TT/MPS rank when seen as a tensor. This typically arises from a discretization of a high-dimensional operator on a tensor grid. In our numerical experiments we have taken the $M$-dimensional Laplace operator with Dirichlet boundary conditions, discretized by the usual second-order central finite difference on a uniform grid with $2^{d}$ points in each mode. 
As a low-rank format, we used the quantized TT-format (QTT) $[25,14]$ which coincides with a $M d$-dimensional TT/MPS format with all dimensions $n_{i}=2$. It is known [13] that in this format the matrix $A$ is represented with QTT-ranks bounded by 4 . Since $A$ is symmetric positive definite, as an initial guess we choose $Y_{0}=\alpha I$ with a sufficiently small $\alpha$. The splitting integrator is applied with $\Delta_{k}=Y_{k}-Y_{k} A Y_{k}$. It requires a certain amount of technical work to implement all the operations involved in the QTT format, but the final complexity is linear in the dimension of the tensor (but of course, has high polynomial complexity with respect to the rank). To put the solution onto the right manifold we artificially add a zero tensor to the initial guess, which has rank 1, and formally apply the splitting integrator.

As first numerical result, we compare the projector-splitting scheme to the standard approach where after each step of the Newton-Schultz iteration we project onto a manifold of tensors with bounded TT/MPS ranks $r$ using the TT-SVD,

$$
Y_{k+1}=P_{r}\left(2 Y_{k}-Y_{k} A Y_{k}\right) \text {. }
$$

The parameters are set as $M=2, d=7, r=20, \alpha=10^{-2}$. The convergence of the relative residual $\left\|A Y_{k}-I\right\| /\left\|A Y_{0}-I\right\|$ in the Frobenius norm for the two methods is presented in Fig. 6.2. The splitting method has slightly better accuracy and, more importantly, is significantly faster.

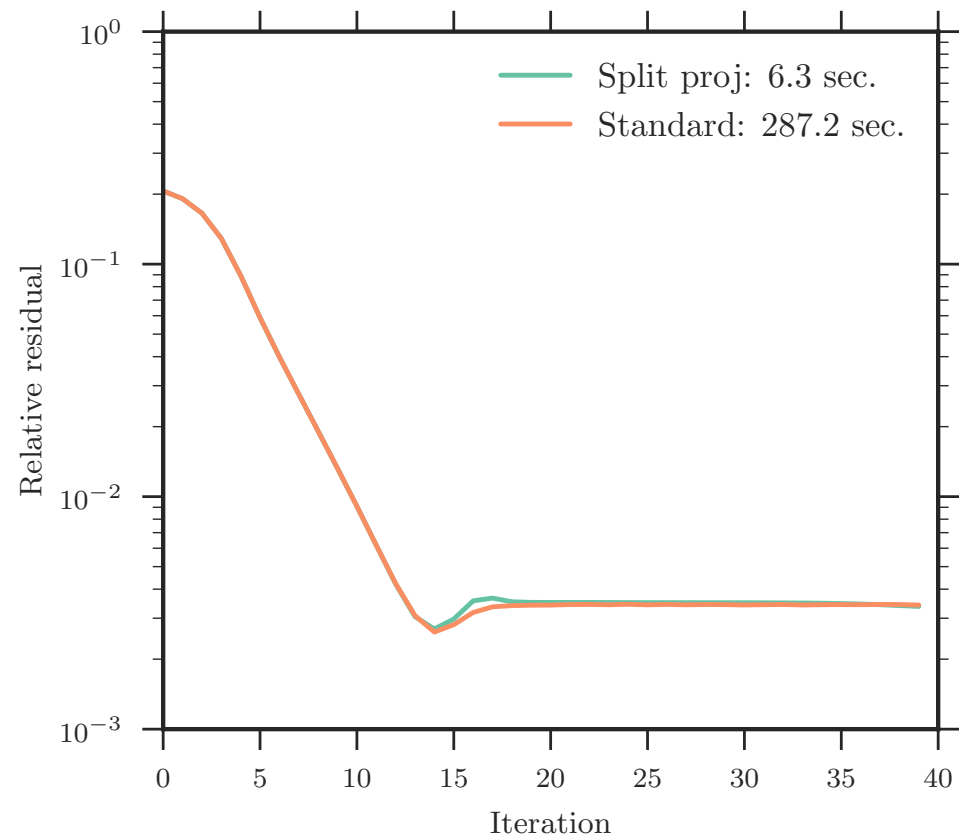

FIG. 6.2. Convergence of split projector method and the SVD-based projection method for $D=2, d=7, \alpha=10^{-2}, r=20$

During the numerical experiments we observed that the residual always decreases until the point when the manifold is insufficient to hold a good approximation to an inverse, and then it either stabilizes or diverges. The exact explanation of this behavior is out of the scope of the current paper but could probably be solved using a proper line-search on $\mathcal{M}$ as in [1]. Fig. 6.3 shows the convergence behavior for 


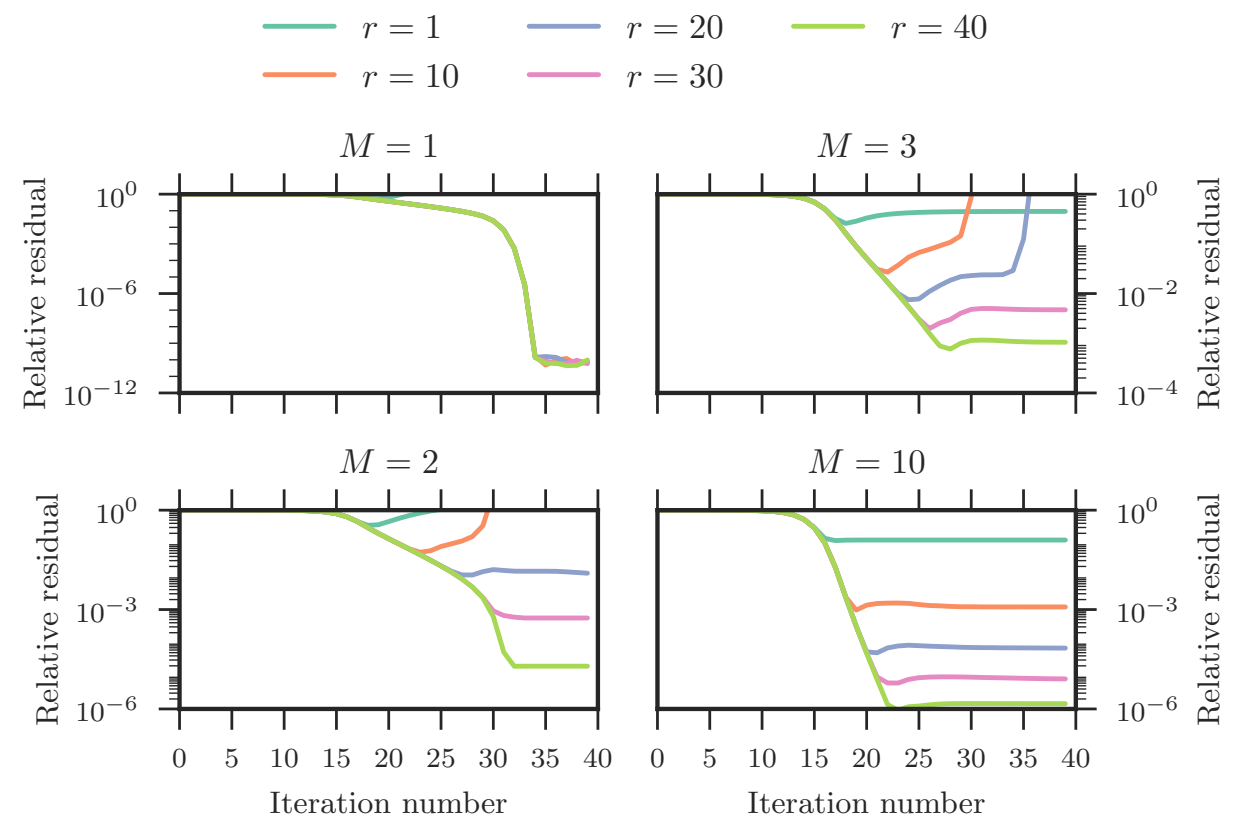

FIG. 6.3. The relative residual vs. iteration number for the approximate inversion using $T T / M P S$ rank $r$ of the $M$-dimensional Laplace operator on a uniform grid with $2^{7}=128$ points in each dimension. Fixed starting guess $Y_{0}=\alpha I$ with $\alpha=10^{-6}$.

different $M$ and $r$, with $d$ and $\alpha$ fixed. Fig. 6.4 shows the convergence behavior with respect to different $\alpha$ and $d$. Finally, Fig. 6.5 shows that the code has good scaling with $d$ and $M$.

7. Conclusion. We have presented and studied a robust and computationally efficient integrator for updating tensors in the tensor train or matrix product state format and for approximately solving tensor differential equations with the approximations retaining the data-sparse tensor train format. Quantum dynamics and tensor optimization appear as promising application areas.

It appears possible to extend this approach to the manifold of hierarchical Tucker tensors of fixed rank [31] and its dynamical approximation [20]. This will be reported elsewhere. In addition, the integrator shares a close resemblance to alternating least squares (ALS) or one-site DMRG (see, e.g., [11, 4] and for a geometric analysis [28]) when the time step goes to infinity. This requires further investigation.

Acknowledgement. We thank Jutho Haegeman and Frank Verstraete (Gent) for helpful discussions regarding matrix product states and the splitting integrator, and Hans-Dieter Meyer (Heidelberg) for explaining the basic concepts behind quantum molecular dynamics simulations and for his help with the MCTDH package.

We thank the two referees as well as Emil Kieri (Uppsala) and Hanna Walach (Tübingen) for pointing out numerous typos in a previous version and for suggesting improvements of the presentation.

The work of C.L. was supported by DFG through SPP 1324 and GRK 1838. The work of I.O. was supported by Russian Science Foundation grant 14-11-00659. 


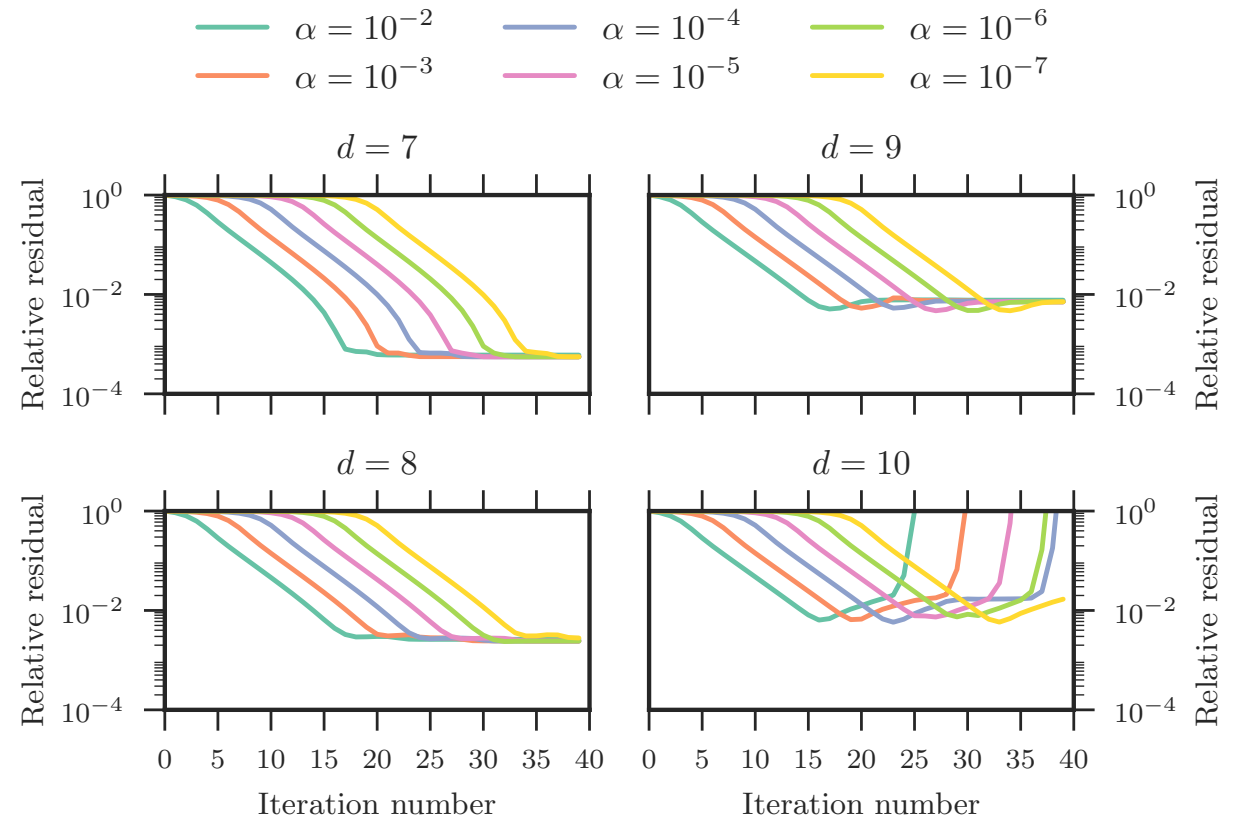

FIG. 6.4. The relative residual vs. iteration number for the approximate inversion using TT/MPS rank 30 of the 2-dimensional Laplace operator a uniform grid with $2^{d}$ points in each dimension. Starting guesses are $Y_{0}=\alpha I$.

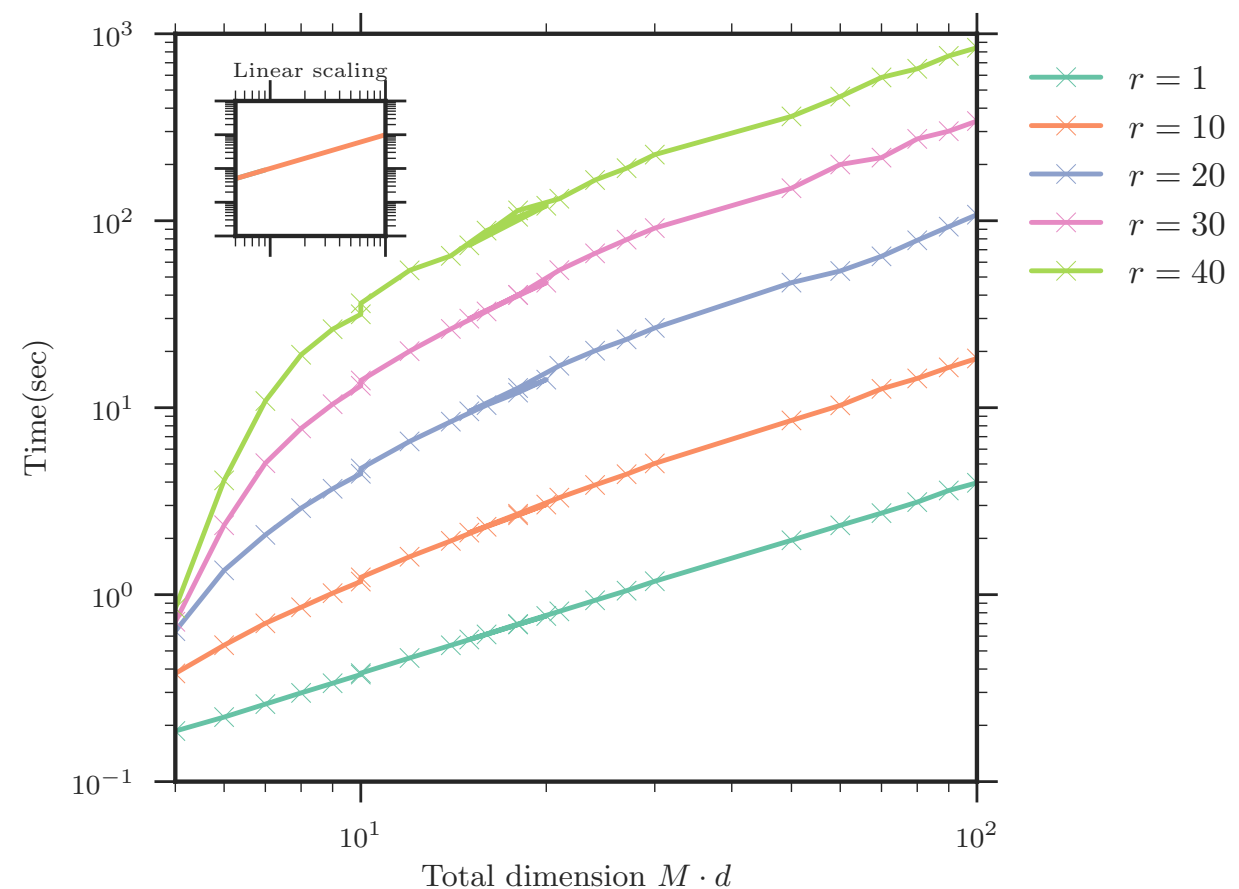

FIG. 6.5. Time in log-log scale as a function of the total dimension Md of the tensor 


\section{REFERENCES}

[1] P.-A. Absil, R. Mahony, and R. Sepulchre, Optimization Algorithms on Matrix Manifolds, Princeton University Press, Princeton, NJ, 2008.

[2] M. H. Beck, A. Jäckle, G. A. Worth, and H.-D. Meyer, The multiconfiguration timedependent Hartree method: A highly efficient algorithm for propagating wavepackets., Phys. Rep., 324 (2000), pp. 1-105.

[3] D. T. Colbert And W. H. Miller, A novel discrete variable representation for quantum mechanical reactive scattering via the S-matrix Kohn method, J. Chem. Phys., 96 (1992), pp. 1982-1991.

[4] S. V. Dolgov And I. V. Oseledets, Solution of linear systems and matrix inversion in the TT-format, SIAM J. Sci. Comput., 34 (2012), pp. A2718-A2739.

[5] W. Hackbusch, Tensor spaces and numerical tensor calculus., Berlin: Springer, 2012.

[6] W. Hackbusch, B. Khoromskis, and E. Tyrtyshnikov, Approximate iterations for structured matrices, Numer. Math., 109 (2008), pp. 365-383.

[7] J. Haegeman, T. J. Osborne, and F. Verstraete, Post-matrix product state methods: To tangent space and beyond, Phys. Rev. B, 88 (2013), p. 075133.

[8] E. Hairer, C. Lubich, and G. Wanner, Geometric Numerical Integration, Springer-Verlag, Berlin, Germany, second ed., 2006.

[9] M. HochBruCK AND C. LuBICH, On Krylov subspace approximations to the matrix exponential operator, SIAM J. Numer. Anal., 34 (1997), pp. 1911-1925.

[10] M. Hochbruck and A. Ostermann, Exponential integrators, Acta Numerica, 19 (2010), pp. 209-286.

[11] S. Holtz, T. Rohwedder, And R. Schneider, The alternating linear scheme for tensor optimisation in the TT format, SIAM J. on Sci. Comput., 34 (2012).

[12] —, On manifolds of tensors of fixed TT-rank, Numer. Math., 120 (2012), pp. 701-731.

[13] V. A. Kazeev and B. N. KhoromskiJ, Low-rank explicit QTT representation of the Laplace operator and its inverse, SIAM J. Matrix Anal. Appl., 33 (2012), pp. 742-758.

[14] B. N. KhoromskiJ, $\mathcal{O}(d \log n)$-Quantics approximation of $N$-d tensors in high-dimensional numerical modeling, Constr. Approx., 34 (2011), pp. 257-280.

[15] O. Koch AND C. LUBICH, Dynamical low-rank approximation, SIAM J. Matrix Anal. Appl., 29 (2007), pp. 434-454.

[16] T. G. KOLDA AND B. W. BADER, Tensor decompositions and applications, SIAM Review, 51 (2009), pp. 455-500.

[17] P. Kramer and M. Saraceno, Geometry of the time-dependent variational principle in quantum mechanics, vol. 140 of Lecture Notes in Physics, Springer-Verlag, Berlin-New York, 1981.

[18] C. Lubich, From quantum to classical molecular dynamics: reduced models and numerical analysis, Zurich Lectures in Advanced Mathematics, European Mathematical Society (EMS), Zürich, 2008.

[19] C. Lubich and I. Oseledets, A projector-splitting integrator for dynamical low-rank approximation, BIT, 54 (2014), pp. 171-188.

[20] C. Lubich, T. Rohwedder, R. Schneider, and B. Vandereycken, Dynamical approximation of hierarchical Tucker and tensor-train tensors, SIAM J. Matrix Anal. Appl., 34 (2013), pp. 470-494.

[21] U. Manthe, H.-D. Meyer, and L. S. Cederbaum, Wave-packet dynamics within the multiconfiguration Hartree framework: General aspects and application to $\mathrm{NOCl}$, J. Chem. Phys., 97 (1992), pp. 3199-3213.

[22] H.-D. Meyer, F. Gatti, And G. A. Worth, eds., Multidimensional Quantum Dynamics: MCTDH Theory and Applications, Wiley-VCH, Weinheim, 2009.

[23] H.-D. Meyer And G. A. Worth, Quantum molecular dynamics: Propagating wavepackets and density operators using the multiconfiguration time-dependent Hartree (MCTDH) method, Theor. Chem. Acc., 109 (2003), pp. 251-267.

[24] M. Nest And H.-D. Meyer, Benchmark calculations on high-dimensional Henon-Heiles potentials with the multi-configuration time dependent Hartree (MCTDH) method, J. Chem. Phys., 117 (2002), p. 10499.

[25] I. V. Oseledets, Approximation of $2^{d} \times 2^{d}$ matrices using tensor decomposition, SIAM J. Matrix Anal. Appl., 31 (2010), pp. 2130-2145.

[26] —, Tensor-train decomposition, SIAM J. Sci. Comput., 33 (2011), pp. 2295-2317.

[27] I. V. Oseledets And E. E. Tyrtyshnikov, Breaking the curse of dimensionality, or how to use SVD in many dimensions, SIAM J. Sci. Comput., 31 (2009), pp. 3744-3759.

[28] T. Rohwedder AND A. Uschmajew, On local convergence of alternating schemes for opti- 
mization of convex problems in the tensor train format, SIAM J. Numer. Anal., 5 (2013), pp. 1134-1162.

[29] U. SCHOLLWÖCK, The density-matrix renormalization group in the age of matrix product states, Annals of Physics, 326 (2011), pp. 96-192.

[30] R. B. SiDJE, Expokit: a software package for computing matrix exponentials, ACM Transactions on Mathematical Software (TOMS), 24 (1998), pp. 130-156.

[31] A. Uschmajew and B. Vandereycken, The geometry of algorithms using hierarchical tensors, Lin. Alg. Appl., 439 (2013), pp. 133-166.

[32] G. A. Worth, M. H. Beck, A. Järkle, And H.-D. Meyer. The MCTDH Package, Version 8.2, (2000). H.-D. Meyer, Version 8.3 (2002), Version 8.4 (2007). See http://mctdh.uni-hd.de. 